\title{
TPL2 enforces $R A S$-induced inflammatory signaling and is activated by point mutations
}

\author{
Paarth B. Dodhiawala, ${ }^{1,2}$ Namrata Khurana, ${ }^{1,2}$ Daoxiang Zhang, ${ }^{1,2}$ Yi Cheng, ${ }^{1,2}$ Lin Li, ${ }^{1,2}$ Qing Wei, ${ }^{1,2,3}$ Kuljeet Seehra, ${ }^{1,2}$ \\ Hongmei Jiang, ${ }^{1,2}$ Patrick M. Grierson, ${ }^{1,2}$ Andrea Wang-Gillam, ${ }^{1,2}$ and Kian-Huat Lim ${ }^{1,2}$ \\ 'Division of Oncology, Department of Internal Medicine, and ²Alvin J. Siteman Comprehensive Cancer Center, Washington University School of Medicine, St. Louis, Missouri, USA. ${ }^{3}$ Department of Laboratory \\ Medicine, Renji Hospital, School of Medicine, Shanghai Jiaotong University, Shanghai, China.
}

\begin{abstract}
$\mathrm{NF}-\kappa \mathrm{B}$ transcription factors, driven by the IRAK/IKK cascade, confer treatment resistance in pancreatic ductal adenocarcinoma (PDAC), a cancer characterized by near-universal KRAS mutation. Through reverse-phase protein array and RNA sequencing we discovered that IRAK4 also contributes substantially to MAPK activation in KRAS-mutant PDAC. IRAK4 ablation completely blocked RAS-induced transformation of human and murine cells. Mechanistically, expression of mutant KRAS stimulated an inflammatory, autocrine IL-1 $\beta$ signaling loop that activated IRAK4 and the MAPK pathway. Downstream of IRAK4, we uncovered TPL2 (also known as MAP3K8 or COT) as the essential kinase that propels both MAPK and NF-KB cascades. Inhibition of TPL2 blocked both MAPK and NF-KB signaling, and suppressed KRAS-mutant cell growth. To counter chemotherapy-induced genotoxic stress, PDAC cells upregulated TLR9, which activated prosurvival IRAK4/TPL2 signaling. Accordingly, a TPL2 inhibitor synergized with chemotherapy to curb PDAC growth in vivo. Finally, from TCCA we characterized 2 MAP3K8 point mutations that hyperactivate MAPK and NF- $\kappa B$ cascades by impeding TPL2 protein degradation. Cancer cell lines naturally harboring these MAP3K8 mutations are strikingly sensitive to TPL2 inhibition, underscoring the need to identify these potentially targetable mutations in patients. Overall, our study establishes TPL2 as a promising therapeutic target in RAS- and MAP3K8-mutant cancers and strongly prompts development of TPL2 inhibitors for preclinical and clinical studies.
\end{abstract}

\section{Introduction}

Targeting the RAS oncoproteins remains unfulfilled in the clinic. Although a newly developed KRAS ${ }^{\mathrm{G} 12 \mathrm{C}}$ inhibitor has achieved considerable success in lung cancer (1), it is ineffective in other $K R A S^{G 12 C}$-mutant cancer types such as colon cancer. In addition, HRAS, NRAS, and non-G12C KRAS oncoproteins remain undruggable. In pancreatic ductal adenocarcinoma (PDAC), though KRAS mutations are virtually universal, the G12C mutation is rare (2). Strategies to target KRAS effectors including the mitogenactivated protein kinase (MAPK) and phosphoinositide $\underline{3}$-kinase (PI3K) cascades are unsuccessful (3) and multiple resistance mechanisms have been described explaining their failures. Aberrant activation of the NF- $\kappa \mathrm{B}$ transcription factors, especially the RELA (or p65) family member, occurs in approximately two-thirds of PDAC and is a major mechanism that underlies the aggressive nature of PDAC (4-7). In a genetically-engineered mouse model (GEMM), ablation of IKK $\beta$ kinase, which activates the NF- $\kappa B$ members, completely abolished KRAS ${ }^{G 12 D}$-induced PDAC development (8). However, development of IKK inhibitors is hampered by clinical toxicities and off-target effects (9). PDAC cells and the surrounding stromal fibroblasts secrete IL-1 $\beta$ that engages interleukin-1 receptor-associated kinase 4 (IRAK4) to drive IKK $\beta$ and the NF- $\kappa B$ pathway (10), indicating that IRAK4 is a promising ther-

Conflict of interest: The authors have declared that no conflict of interest exists. Copyright: () 2020, American Society for Clinical Investigation.

Submitted: March 2, 2020; Accepted: June 10, 2020; Published: August 10, 2020.

Reference information: J Clin Invest. 2020;130(9):4771-4790.

https://doi.org/10.1172/JCl137660. apeutic target. IRAK4 is a critical signal transducer downstream of the innate immune receptors including the Toll-like (TLR) and IL-1 (IL-1R) receptors (11). When engaged, these receptors recruit MYD88 and IRAK1 as adaptors, forming a platform that recruits IRAK4. IRAK4 then activates the IKK complex, allowing cytoplasmic NF- $\kappa$ B subunits such as RELA/p65 and p50 to enter the nucleus and transactivate inflammatory and survival genes (12). The protumorigenic role of this pathway has been described in melanoma (13), breast (14), head and neck (15), colon (16), and pancreatic cancers $(7,10)$. However, these studies do not describe the genetic context in which IRAK4 inhibition is most likely to succeed, nor do they provide insights into the crosstalk of IRAK4 signaling with other oncogenic events besides the NF- $\mathrm{B}$ pathway.

In this study, we interrogated The Cancer Genome Atlas (TCGA) database and found that MYD88, IRAK1, and IRAK4 are associated with RELA expression and poor prognosis in PDAC. We further found that IRAK4 is essential for RAS-induced oncogenic transformation. Interestingly, by unbiased reverse-phase protein array (RPPA) and RNA sequencing we discovered signaling crosstalk between IRAK4 and the MAPK pathway in KRAS-mutant PDAC cells. We elucidate the mechanism of how oncogenic KRAS activates IRAK4, and uncover tumor progression locus 2 (TPL2, also known as MAP3K8 or COT) as the essential kinase that controls both MAPK and NF- $\mathrm{BB}$ cascades downstream of IRAK4 and effectively KRAS. In addition, we interrogate the role of TPL2 under genotoxic stress and show that a TPL2 inhibitor (TPL2i) synergizes with the FIRINOX chemotherapy regimen to suppress human and murine in vivo PDAC growth. Last, we screened recurrent MAP3K 8 mutations from TCGA and discovered 2 gain-of- 
A
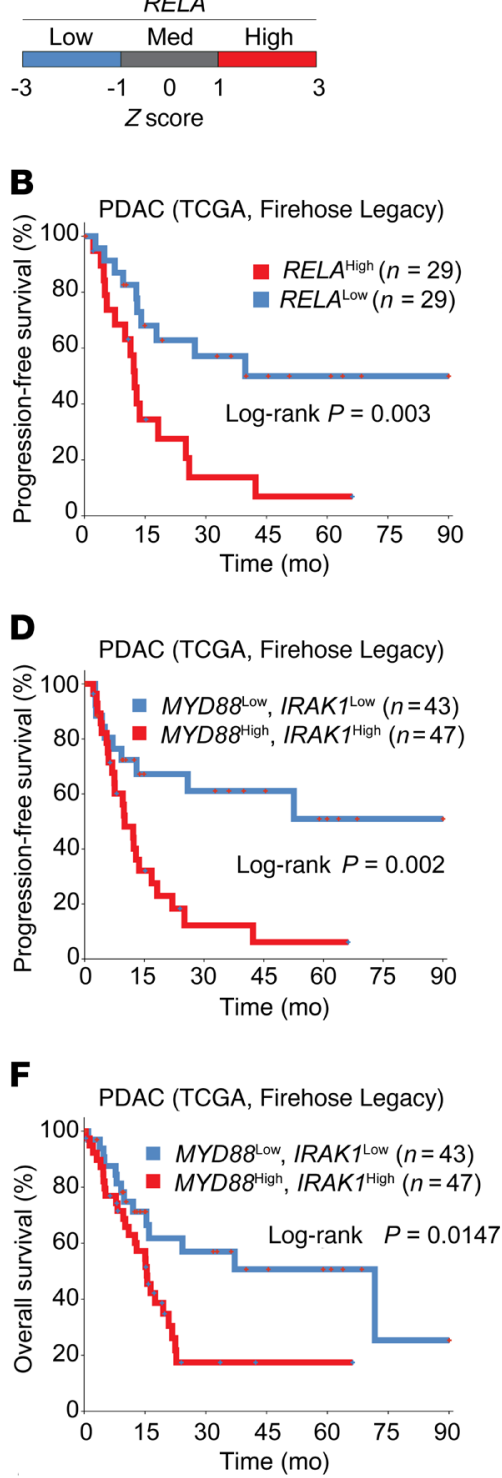

$\mathbf{J}$

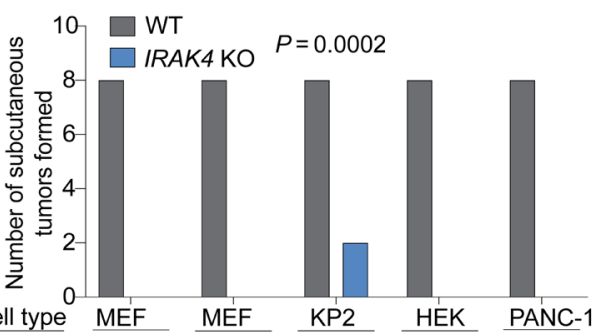

Cell type $\frac{M E F}{M E F} \frac{\text { KP2 }}{\text { MEF }} \frac{\text { HEK }}{\text { PANC-1 }}$

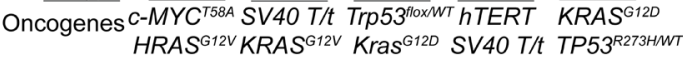
KRAS ${ }^{\mathrm{G} 12 \mathrm{~V}}$
PDAC (TCGA, Firehose Legacy)

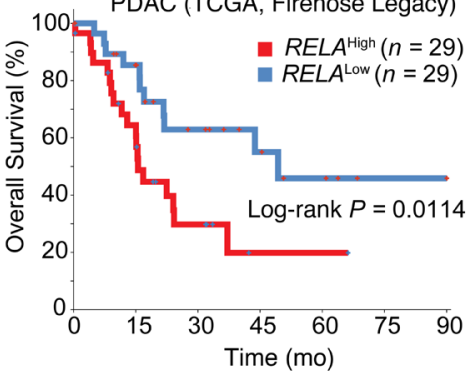

E

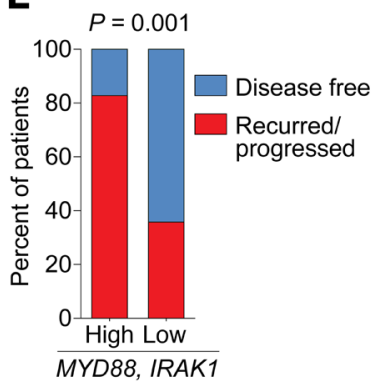

G

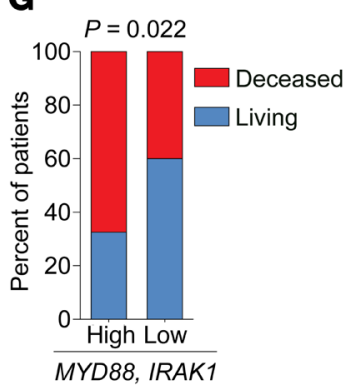

H

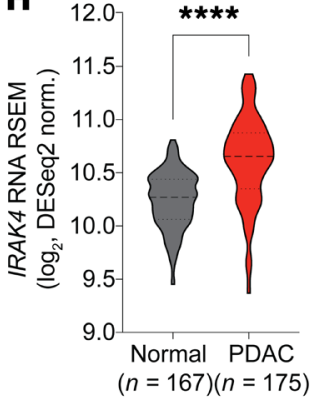

KP2

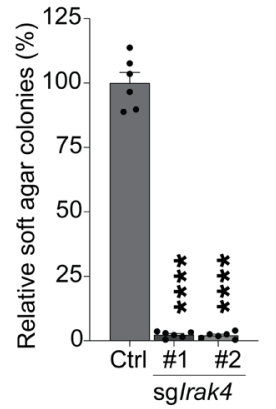

C

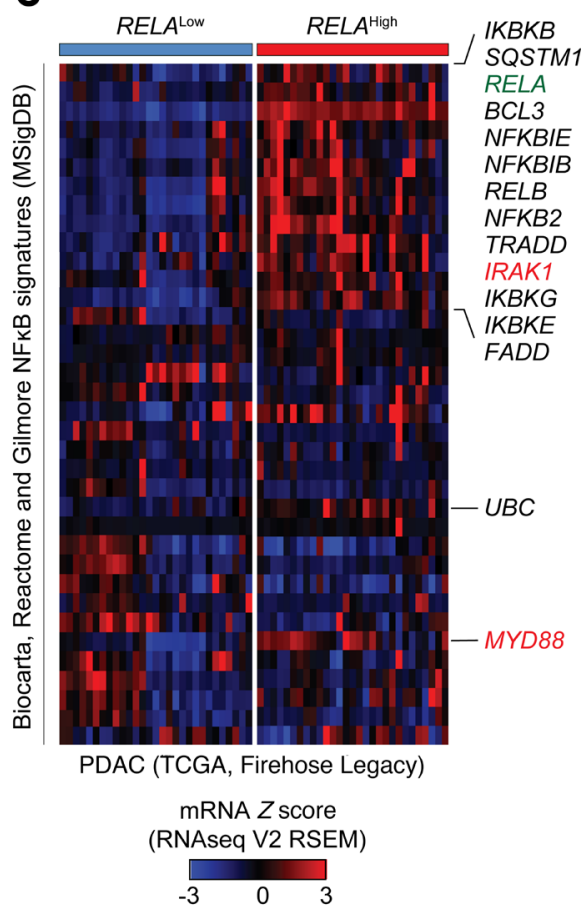

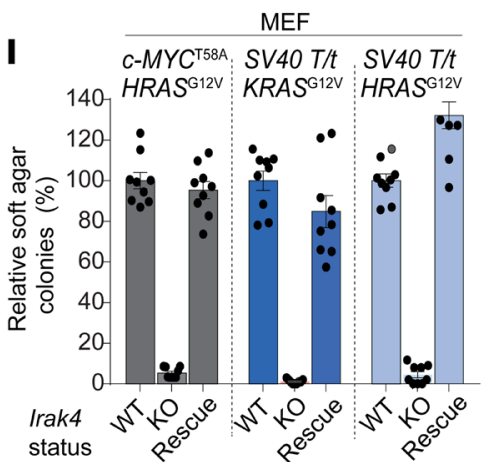

status
$\mathbf{M}$

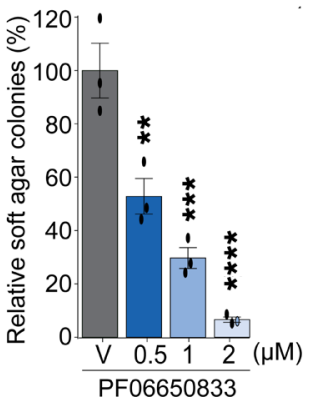

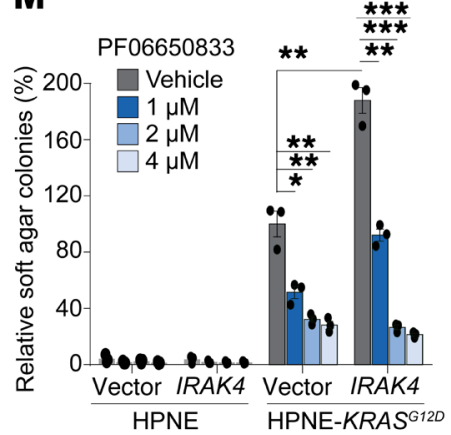


Figure 1. IRAK signaling dictates NF- $\kappa B$ activity in PDAC and is essential for RAS oncogenesis. (A) Classification of patients with low, medium, and high RELA expression based on mRNA Z score from TCGA. (B) Progression-free survival (PFS) and overall survival (OS) of PDAC patients with high versus low RELA expression. (C) Heatmap comparing mRNA expression of NF- $\kappa \mathrm{B}$ signature genes from the Broad Institute Molecular Signatures Database (gene sets are listed in Supplemental Table 3 ) in $R E L A^{\text {High }}$ vs. RELA $A^{\text {Low }}$ patients. ( $\mathbf{D}$ and $\left.\mathbf{E}\right) \mathrm{PFS}$ and disease-free status, respectively, of PDAC patients with high MYD88 and/or high IRAK1 expression ("MYD88 $8^{\text {High }}$, IRAK $1^{\text {High") }}$ versus low MYD88 and/or low IRAK1 expression ("MYD88 $8^{\text {Low }}$, IRAK $1^{\text {Low") }}$. Eight patients overlapping between 2 groups were excluded. ( $\mathbf{F}$ and $\mathbf{G}$ ) $\mathrm{OS}$ and vital status of patients as in $\mathbf{D}$ and E. (H) Graph of IRAK4 expression in normal human pancreas versus PDAC. Data for normal tissue is from the Genotype-Tissue Expression (GTEx) project and PDAC expression from TCCA PanCancer Atlas. $P$ values are from unpaired 2-sided $t$ test. (I) Soft-agar colonies formed by Irak4-KO and rescue $\left(K O+I r a k 4^{W T}\right)$ MEF cells transformed with 3 pairs of oncogenes. Data show 9 replicates from 3 independent experiments. (J) Number of tumors formed in nude mice from subcutaneous implantation of WT and IRAK4KO human and murine RAS-mutant and/or PDAC cell lines. $n=8$ tumors per condition. (K) Quantification of soft-agar colonies formed by WT versus Irak4-KO KP2 cells. Data show 6 replicates from 2 independent experiments. (L) Soft-agar colonies formed by KP2 cells treated with IRAK4i or vehicle (V). (M) Soft-agar colonies formed by WT and KRAS ${ }^{1120}$ HPNE cells ectopically expressing WT IRAK4 and treated with IRAK4i. For $\mathbf{L}$ and M, 3 independent experiments were performed, each in technical triplicate, and one set of data is shown. All error bars indicate mean $\pm \mathrm{SEM}$. ${ }^{* * *} P<$ $0.0001 ;{ }^{* * *} P<0.0002$; ${ }^{* *} P<0.0021 ;{ }^{*} P<0.0332$.

function point mutants, which naturally exist in ovarian cancer and melanoma cell lines, and are highly sensitive to TPL 2 blockade.

\section{Results}

IRAK signaling dictates $N F-\kappa B$ activity in PDAC and is essential for RAS oncogenesis. Aberrant NF- $\mathrm{BB}$ activation is conventionally defined by increased RELA (or p $65 \mathrm{NF}-\kappa \mathrm{B}$ family member) expression or nuclear translocation by immunohistochemistry (IHC) in tumor samples $(17,18)$. To more comprehensively understand the NF- $\mathrm{KB}$-associated transcriptomes in PDAC, we evaluated the expression pattern of 37 core NF- $\kappa \mathrm{B}$ genes, which include RELA as defined by the Molecular Signatures Database (MSigDB) (19), in PDAC samples from TCGA (Firehose Legacy, $n=185$ ). By unsupervised clustering we observed heterogeneous expression of these 37 genes across all samples, reflecting the complexity of mechanisms that activate NF- $\mathrm{BB}$ and presence of different categories of NF- $\kappa \mathrm{B}$ signatures in PDAC (Supplemental Figure 1A; supplemental material available online with this article; https:// doi.org/10.1172/JCI137660DS1). Because RELA expression is the single, most established NF- $\kappa \mathrm{B}$ marker associated with poor prognosis in PDAC, we divided these samples into 3 groups based on $R E L A$ mRNA level: $R E L A^{\text {High }}(Z$ score $>1.0, n=29), R E L A^{\text {Med }}(Z$ score between -1.0 and 1.0, $n=129)$, and $R E L A^{\text {Low }}(Z$ score $<-1.0$, $n=29$ ) (Figure $1 \mathrm{~A}$ ). Compared with the $R E L A^{\text {Low }}$ group, patients in the $R E L A^{\text {High }}$ and $R E L A^{\text {Med }}$ groups had significantly worse progression-free and overall survival (Figure 1B and Supplemental Figure 1B). We focused our analysis on RELA $A^{\text {High }}$ and RELA ${ }^{\text {Low }}$ cohorts in order to discern the remaining NF- $\mathrm{KB}$ genes that cluster with RELA expression. Notably, many genes in the canonical $\mathrm{NF}-\kappa \mathrm{B}$ pathway were upregulated in the $R E L A^{\text {High }}$ group (Figure $1 C)$, such as the IKK isoform genes (IKBKB, IKBKE, and IKBKG),

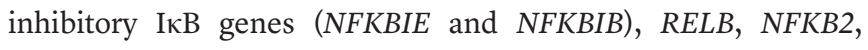
IRAK1, and MYD88. Of these, MYD88 and IRAK1 caught our attention because these are known upstream activators of the IKK kinases (20, 21). MYD88 and IRAK1 expression positively correlated with each other (Supplemental Figure 1C) and RELA (Supplemental Figure 1D). Notably, patients with high ( $Z$ score > 1.0) MYD88 and/or IRAK1 expression (termed "MYD88 8 "igh, IRAK $1^{\text {High") }}$ had significantly worse progression-free survival (log-rank $P=0.0020$, Figure 1D), disease-free status (Figure 1E), overall survival (log-rank $P=0.0147$, Figure $1 \mathrm{~F}$ ), and vital status at the time of data cutoff (Figure $1 \mathrm{G}$ ), compared with patients with low $(Z$ score $<1.0)$ MYD88 and/or IRAK1 expression (termed

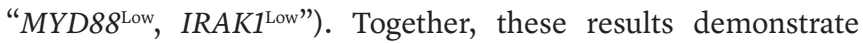
positive correlation between MYD88-IRAK and RELA expression, and further support the TLR/IL-1R canonical pathway as the main driving mechanism of $\mathrm{NF}-\kappa \mathrm{B}$ activity in PDAC that translates into clinical aggressiveness, congruent with published studies $(7,8,10,22)$.

Unlike MYD88 and IRAK1, which function as adaptor proteins, IRAK4 is the bone fide kinase that initiates NF- $\kappa B$ signaling and can be targeted $(7,20)$. Phosphoactivation of IRAK4 is associated with higher RELA activity and poor survival of PDAC patients (7). IRAK4, though not included in the predefined 37-gene MSigDB $\mathrm{NF}-\kappa \mathrm{B}$ signature, was significantly overexpressed in PDAC compared with normal pancreas (Figure $1 \mathrm{H}$ ). Analysis of the whole TCGA data showed significantly higher IRAK4 expression in PDAC compared with majority of other cancers (Supplemental Figure 1E and Supplemental Table 1), supporting a pathogenic role of IRAK4 in PDAC.

Both IRAK4 and KRAS can drive NF- $\kappa \mathrm{B}$ signaling in PDAC, but their crosstalk has not been investigated. While KRAS oncoprotein can stimulate the noncanonical IKK through the RalGEF/ RalB/TBK1 effector (23) and the canonical IKK $\alpha / \beta$ through the PI3K/AKT/mTOR effectors (24), IRAK4 uses TAK1 kinase to activate IKK $\beta$ (12). Therefore, IRAK4 should not be required for KRAS-induced oncogenesis per se. Yet, to test this, we stably expressed pairs of oncogenes including $c-M Y C^{T 58 A}$ and $H R A S^{G 12 V}$, SV-40 T/t antigens and HRAS ${ }^{G 12 V}$, or SV-40 T/t and KRAS ${ }^{G 12 V}$ in $\mathrm{WT}$ and $\mathrm{Irak}^{--}$murine embryonic fibroblasts (MEFs). Surprisingly, anchorage-independent (AI) growth in soft agar, a classical assay for transformation, is completely abrogated in IRAK4 $4^{-/}$ MEFs, but this could be fully rescued with reexpression of murine Irak4 cDNA (Figure 1I). Consistently, Irak4 ${ }^{--}$MEFs expressing oncogenic HRAS or KRAS failed to form tumors in nude mice (Figure 1J). As further confirmation, we employed the CRISPR/Cas9 technique to ablate IRAK4 in 3 KRAS-transformed cell lines: KP2 (a murine PDAC line originating from a $p 48$-Cre; Tp5 $3^{f / W T}$; LSL$\operatorname{Kras}^{G 12 D}$, or KPC mouse), a human embryonic kidney (HEK T/tH) line transformed with hTERT, SV-40 T/t, and KRAS G12V, and the $K R A S^{G 12 D}$-mutant PANC-1 line. Again, loss of IRAK4 completely abrogated tumorigenic growth of these lines in nude mice (Figure 1J). KP2 cells expressing single guide RNAs against Irak4 (sgIrak4) were severely impaired not only in AI growth, but also 2-dimensional (2D) proliferation (Figure 1K and Supplemental Figure 1F), revealing a role of IRAK4 in cell fitness. Similarly, AI growth of KP2 cells was dose-dependently suppressed by PF06650833 (25), a selective IRAK4 inhibitor (IRAK4i) Figure 1L). To dissect the role 
A

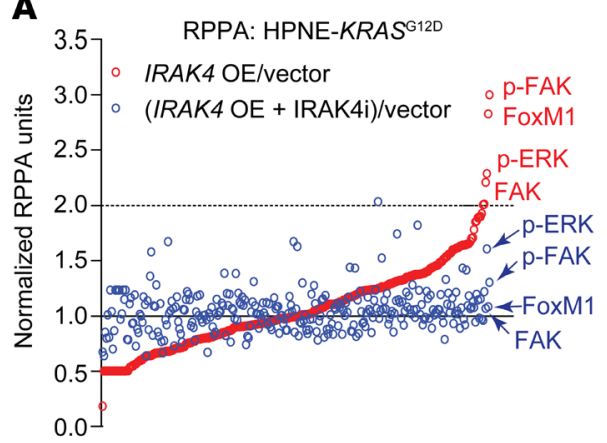

B

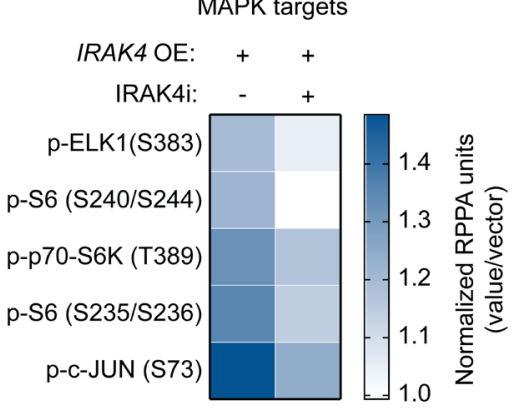

C

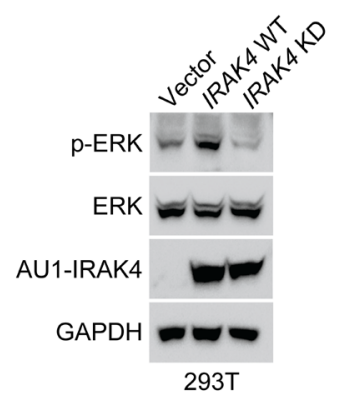

$\mathbf{E}$

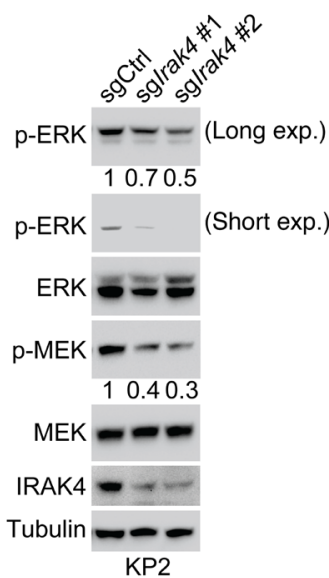

H

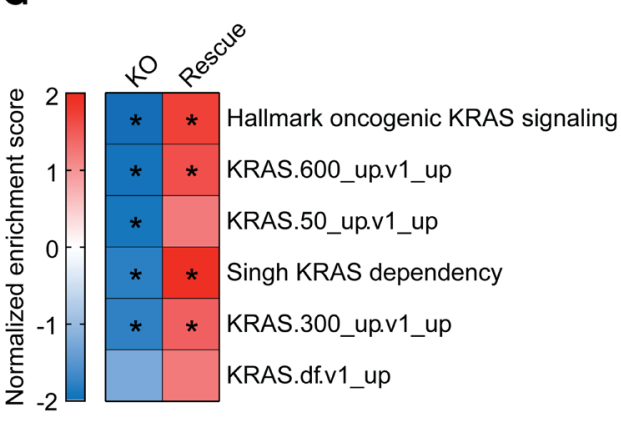

PDAC signature

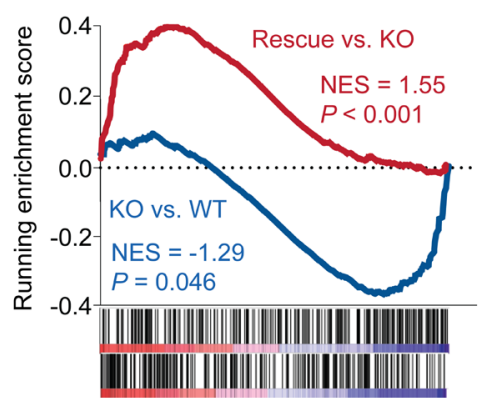

I

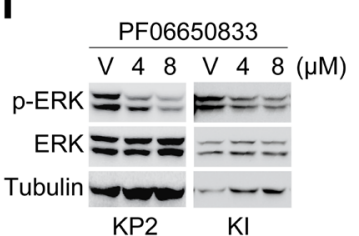

J

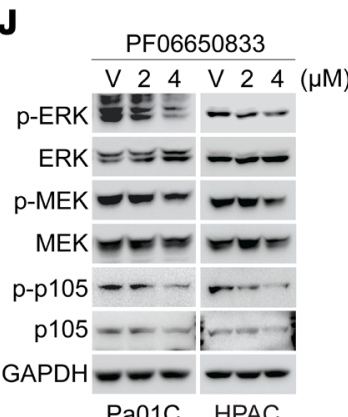

$\mathbf{K}$

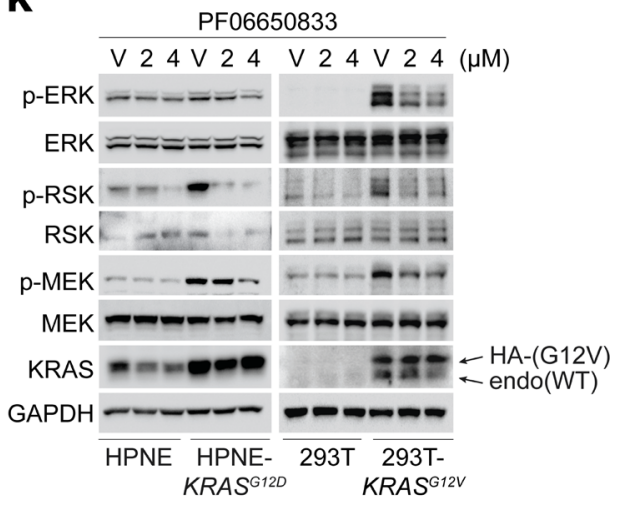


Figure 2. IRAK4 is crucial for oncogenic RAS-driven MAPK signaling.

(A) Linear fold-change for all targets evaluated by reverse-phase protein array (RPPA) performed on HPNE-KRAS 120 overexpressing (OE) IRAK4 and treated with IRAK4i. Targets with fold-change $>2$ upon IRAK4 overexpression are identified. (B) Heatmap showing relative expression of ERK-regulated targets in RPPA shown in A. (C) Immunoblots of 293T cells transfected with AU1 epitope-tagged WT or kinase-dead IRAK4. (D) Heatmap depicting fold-change for MAPK-, RAS-, and cell growth-related Gene Ontology (GO) signatures upon Irak4 knockout (KO) and rescue (KO + Irak4 ${ }^{W T}$ ) in murine KP2 cells. Comparisons are KO vs. WT and rescue vs. KO. Signatures significantly $(P<0.05)$ depleted (blue) or enriched (red) are marked with an asterisk $\left(^{*}\right)$. (E) Immunoblots of WT and Irak4-KO KP2 cells. (F and $\mathbf{G}$ ) Gene set enrichment plot and normalized enrichment scores (NES), respectively, for signatures associated with oncogenic KRAS in WT, Irak4-KO, and rescue KP2 cells, compared as in D. "Hallmark" and "C6: Oncogenic Signatures" databases were used. Negative NES indicates downregulation and signatures significantly $(P<0.05)$ depleted (blue) or enriched (red) are marked with an asterisk $\left.{ }^{*}\right)$. (H) Gene set enrichment plot for PDAC signature in Irak4-KO and -rescue KP2 cells. PDAC signature gene list is provided in Supplemental Table 3. Barcode plots under curves in $\mathbf{F}$ and $\mathbf{H}$ depict the enrichment clustering for individual genes in the respective gene signatures interrogated for KO vs. WT (top barcode) and rescue vs. KO (bottom barcode) cells. (I) Immunoblots of KP2 and KI cells treated with IRAK4i (PF06650833) or vehicle (V) for 24 hours in serum-free condition. (J) Immunoblots of PDAC cells treated with IRAK4i for 16 hours. (K) Immunoblots of HPNE-KRAS ${ }^{1120}$ and 293T-KRAS ${ }^{612 V}$ cells treated with IRAK4i for 24 hours in serum-free media. For $\mathbf{D}$ and $\mathbf{F}-\mathbf{H}$, RNA sequencing was performed on $n=2$ independent samples for each condition.

of IRAK4 in KRAS-mutant human PDAC, we resorted to the HPNE model, an artificial pancreatic ductal epithelial line immortalized with $h T E R T$ and HPV E6/E7. Stable expression of KRAS ${ }^{G 12 D}$ in this line (named HPNE-KRAS ${ }^{G 12 D}$ ) completes the malignant transformation, enabling AI growth. We found that ectopic expression of IRAK4 doubled the AI growth of HPNE-KRAS ${ }^{G 12 D}$ cells but had no effect on the untransformed HPNE cells (Figure 1M). Notably, treatment with PF06650833 not only reversed the effect of IRAK 4 overexpression, but also crippled the AI growth of HPNE$K R A S^{G 12 D}$. Together, these studies showed IRAK4 is essential for and cooperates with mutant $R A S$ in oncogenic transformation.

IRAK4 is crucial for oncogenic RAS-driven MAPK signaling. To understand the mechanism by which IRAK4 promotes $R A S$ induced transformation, we first performed an unbiased RPPA on HPNE-KRAS ${ }^{G 12 D}$ cells stably expressing IRAK4 or empty vector. Compared with vector-expressing cells, IRAK4-overexpressing cells exhibited a greater than 2-fold increase in phosphorylated FAK (p-FAK), p-ERK, total FAK, and FoxM1, which was reversed by IRAK4i treatment (Figure 2A). Indeed, various MAPK target proteins were activated by IRAK4 overexpression and conversely suppressed by IRAK4i (Figure 2B). We observed similar changes with p-p65/RELA (Supplemental Figure 2A), resonating with our previous studies $(7,10)$. As confirmation, overexpression of WT, but not kinase-dead IRAK4, enhanced p-ERK in 293T cells, as determined by Western blotting (Figure 2C). Next, we performed RNA sequencing analysis on KP2 cells expressing vector, 2 different sgIrak4, or sgIrak4 followed by rescue with a murine Irak4 cDNA (termed "rescue"; Supplemental Figure 2B). Consistent with our RPPA results, MAPK, ERK, and progrowth gene ontology (GO) signatures were significantly suppressed in Irak4-depleted KP2 cells and restored in Irak4-rescued cells (Figure 2D). As con- firmation, Western blots showed reduced p-MEK and p-ERK levels in IRAK4-ablated KP2 cells (Figure 2E). Various NF- $\kappa B$ signatures were also similarly affected (Supplemental Figure 2C). Gene set enrichment analysis (GSEA) showed oncogenic KRAS signaling and PDAC signatures to be significantly downregulated following Irak4 ablation and restored in Irak4-rescued cells (Figure 2, F-H). In accordance, IRAK $4 \mathrm{i}$ dose-dependently suppressed MAPK activity in 2 murine PDAC lines, KP2 and KI (derived from a PDX1-Cre; INK $4 a^{f l / f l}$; LSL-KRAS ${ }^{G 12 D}$ mouse), as well as KRAS-mutant PDAC patient-derived cell lines (PDCLs) Pa01C, PaO2C, and Pa03C, and a conventional human pancreatic adenocarcinoma cell line, HPAC (Figure 2, I and J, and Supplemental Figure 2D). As further confirmation, IRAK4i dose-dependently reduced serum-response element-driven (SRE-driven) luciferase reporter activity in HPAC cells (Supplemental Figure 2E) and viability of various $K R A S$ mutant PDAC lines in vitro (Supplemental Figure 2F). To clearly establish the role of IRAK4 in KRAS-induced MAPK signaling, we used 2 isogenic pairs of cell lines expressing empty vector, $K R A S^{G 12 D}$ (for HPNE), or $K R A S^{G 12 V}$ (for 293T). As expected, expression of KRAS mutants clearly upregulated p-MEK, p-ERK, and p-RSK levels, but all these were dose-dependently blocked by IRAK4i (Figure 2K). Importantly, expression of an activated MEK mutant (MEK1$5^{\mathrm{DD}}$ ) rendered HEK T/tH-KRAS $S^{\mathrm{Gl} V}$ cells approximately 3-fold less sensitive to growth inhibition by IRAK4i (Supplemental Figure $2 \mathrm{G}$ ), positioning IRAK4 upstream of MEK. Besides the MAPK pathway, IRAK4i also suppresses p-p105 levels in Pa01C, Pa02C, PaO3C, and HPAC cells (Figure 2J and Supplemental Figure 2D), resonating with previous findings that IRAK4 is a driver of the NF- $\kappa$ B pathway $(7,16)$. Intriguingly, we also observed dose-dependent suppression of SRE activity by IKK inhibitor IMD-0354 in HPAC, suggesting a contribution of IKK to MAPK activity in PDAC cells (Supplemental Figure 2E). Together, these results establish IRAK4 as a significant contributor to KRAS-induced MAPK signaling.

TPL2 mediates signaling between IRAK4 and the MAPK pathway. Next, we investigated the mechanistic link between IRAK4 and MEK. In myeloid cells, IL-1, TNF, or LPS activates MEK and ERK through engaging TPL2 kinase (or COT/MAP3K8) $(26,27)$. We therefore hypothesized that IRAK4 engages TPL2 to activate MEK and ERK. Indeed, ectopic expression of TPL2 in PaO1C and HPAC cells enhanced p-MEK and p-ERK levels, but this effect was blocked by IRAK4i. Notably, the ectopically expressed TPL2 protein existed in an activated state, as determined by an anti-p-TPL2 antibody, and was dose-dependently deactivated by IRAK4i, confirming IRAK4 as the upstream activator of TPL2 (Figure 3A and Supplemental Figure 3A). In support, ectopic expression of IRAK4 upregulated p-TPL2, p-MEK, and p-ERK in 293T cells (Figure 3B).

To strengthen the link between IRAK4, TPL2, and MEK, we next performed leading-edge analysis using 15 published TPL2-associated gene signatures from the Broad Institute MSigDB, including significantly downregulated MAPK-related GO signatures identified in Irak4-ablated KP2 cells (Supplemental Table 3). Ablation of Irak4 decreased TPL2 (encoded by MAP3K8) expression in the majority of gene sets, and importantly, low TPL2 expression was closely associated and clustered with low MEK1 expression (Figure 3C and Supplemental Figure 3B). These data position TPL2 as the signal transducer between IRAK4 and MEK in PDAC. In human PDAC lines, we observed strong, positive cor- 


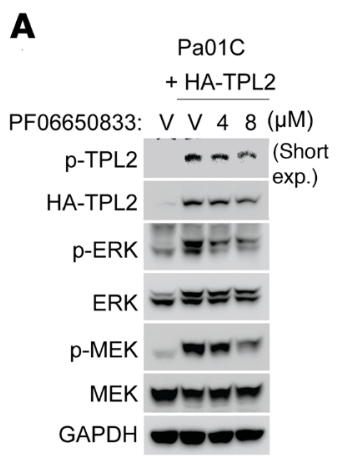

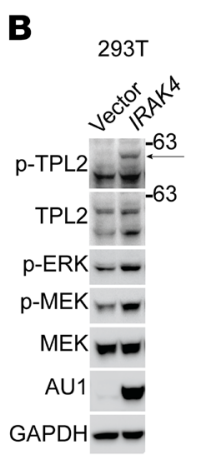

C

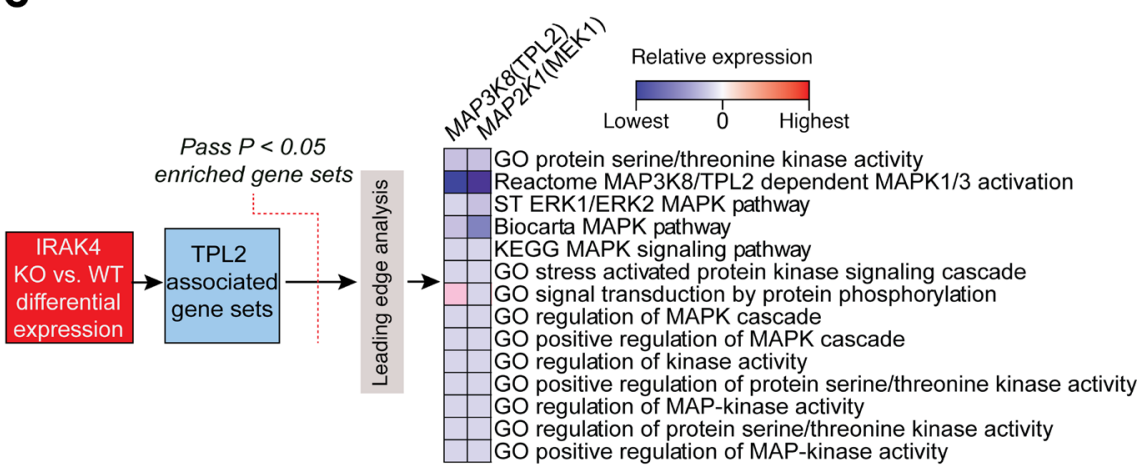

D

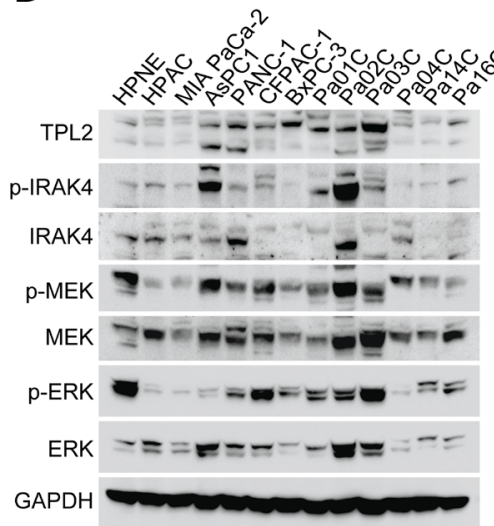

E

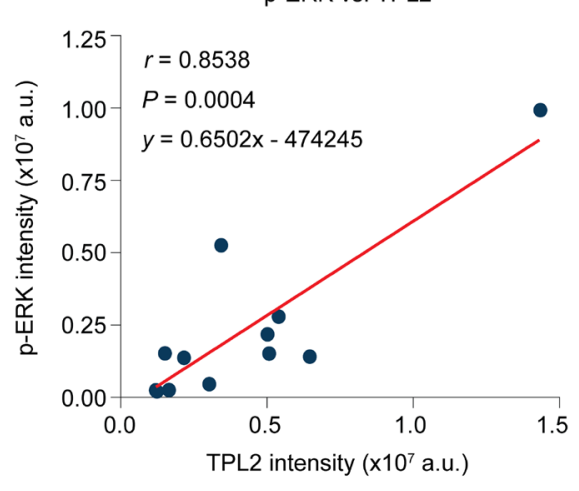

$\mathbf{F}$ $H \& E$

Human

Normal pancreas

DAC
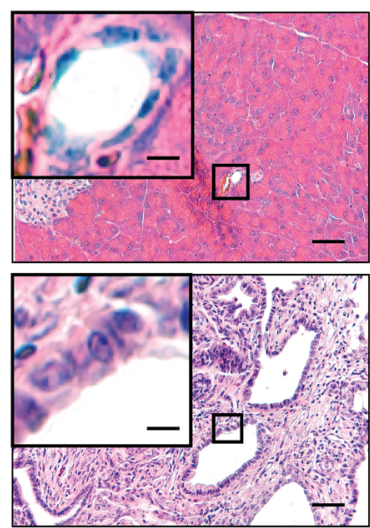

p-IRAK4

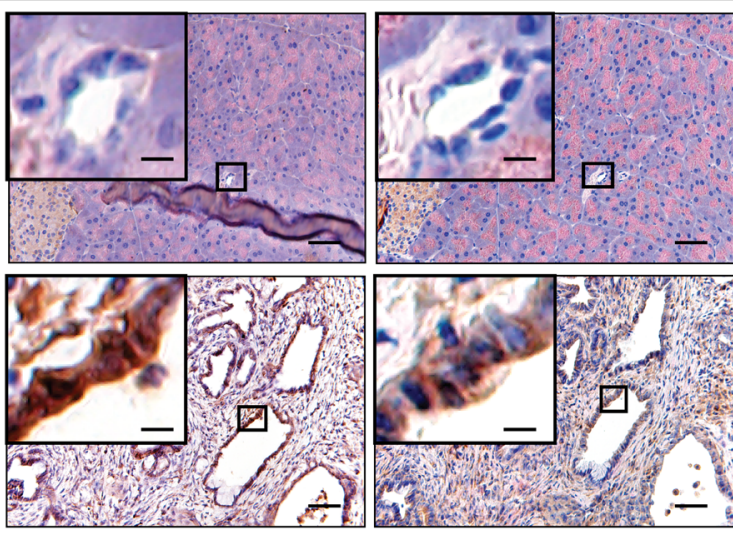

TPL2

p-ERK
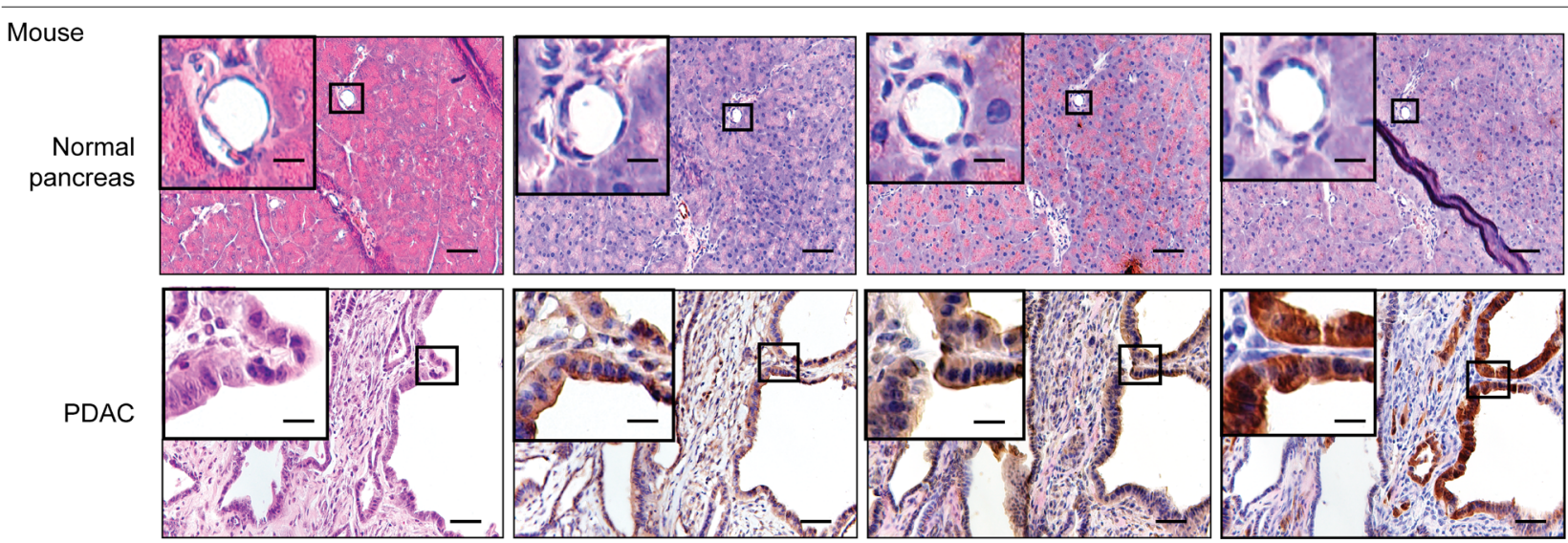
Figure 3. TPL2 mediates signaling between IRAK4 and the MAPK pathway. (A) Immunoblot of Pa01C cells overexpressing HA epitope-tagged TPL2 WT that were treated with IRAK4i or vehicle (V) for 6 hours in serumfree condition. (B) Immunoblots of 293T cells transfected with WT IRAK4 for 48 hours. (C) Leading-edge analysis performed using data generated by gene set enrichment analysis in order to identify alterations in individual genes within each gene set tested. Significantly downregulated $(P<0.05)$ gene signatures were analyzed and a clustered heatmap was generated. Section of heatmap depicting change in MAP3K8 (TPL2) and MAP2K1 (MEK1) expression is shown with original clustering preserved. TPL2-associated gene set list is provided in Supplemental Table 3. (D) Immunoblot of various commercially available and patient-derived (Pa01C-Pa16C) human PDAC cell lines and 1 normal human pancreatic cell line (HPNE). (E) Correlation plot of p-ERK and TPL2 intensities for PDAC cell lines in $\mathbf{D}$. Two-tailed Pearson correlation $(r)$ analysis was performed. (F) Representative H\&E and IHC images of human and murine normal pancreas and PDAC tissue for $p$-ERK, TPL2, and $p$-IRAK4. $n=6$ sections per stain. Scale bars: $50 \mu \mathrm{m}$ (for full image [ $\times 400$ magnification]) and $10 \mu \mathrm{m}$ (insets).

relation between the protein levels of TPL2 and p-ERK (Figure 3, $\mathrm{D}$ and $\mathrm{E})$ by Western blotting. In human and murine PDAC tumor tissues, IHC analyses showed co-occurrence of p-ERK, TPL2, and p-IRAK4 staining in the neoplastic ductal epithelia, whereas these markers were largely absent in normal ductal epithelia (Figure 3F).

High TPL2 expression is poorly prognostic in PDAC. We next performed IHC analyses on a panel of 313 PDAC tissue microarray (TMA) specimens and found strong, significantly positive correlation of $\mathrm{H}$-score, defined by staining area and intensities, between TPL2 and p-IRAK4 $(r=0.56, P<0.0001$; Figure 4 , A and B, and Supplemental Figure 4, A and B). Notably, high TPL2 staining correlated with poor overall survival in PDAC patients based on Wilcoxon's $P=0.03$, a statistic that gives more weight to early deaths, as typically seen with PDAC patients (Figure $4 \mathrm{C}$ ). From TCGA and Genotype-Tissue Expression (GTEx) project databases, MAP3K8 mRNA expression was significantly higher in PDAC compared with normal pancreas (Figure 4D) and a majority of other cancer types (Supplemental Figure 4C and Supplemental Table 2). Next, using the median overall survival of TCGA PDAC patients ( $\sim 15.5$ months), we divided patients into 2 groups, short ( $<15.5$ months overall survival, $n=92$ ) and longer ( $\geq 15.5$ months overall survival, $n=93$ ) survivors (Figure $4 \mathrm{E}$ ). The short survivors had significantly higher MAP3K8 expression (Figure $4 \mathrm{~F}$ ). Conversely, high MAP3K8 expression (arbitrarily defined as $Z$ score $>1, M A P 3 K 8^{\text {High }}, n=22$ ) is significantly associated with poorer overall survival compared with low MAP $3 K 8$ expression ( $Z$ score $<1, M A P 3 K 8^{\text {Low }}, n=28$; Figure $4 G)$. Together, these complementary studies position TPL2 as the intermediate kinase between IRAK4 and MEK and signify its potential as a therapeutic target warranting further investigation.

TPL2 drives both MAPK and NF- $\kappa B$ signaling in PDAC From GSEA analysis we found MAP3K $8^{\text {High }}$ PDAC samples to be enriched for both KRAS and NF- $\mathrm{kB}$ signatures (Figure 5A), mirroring our findings with IRAK4 (Figure 2F) and congruous with a prior report which showed that TPL2 can phosphoactivate the p105 NF- $\mathrm{KB}$ factor (28). To determine if TPL2 controls MAPK and NF- $\mathrm{BB}$ signaling, we treated KRAS-mutant PDAC cells with 4-[(3-chloro-4-fluorophenyl)amino]-6-[(3-pyridinylmethyl) amino]-1,7-naphthyridine-3-carbonitrile, a selective ATP-competitive small-molecule TPL2i that can suppress LPS-induced TNF- $\alpha$ production in human monocytes (29). Treatment with TPL2i dose-dependently suppressed p-MEK and p-ERK levels in multiple KRAS-mutant PDAC lines except PANC-1 (Figure 5B), and in 293T cells expressing KRAS ${ }^{G 12 V}$ (Figure 5C), establishing TPL2 as a contributor to KRAS-induced MAPK activity. Notably, TPL2i dose-dependently blocked p-MEK and p-ERK without affecting p-BRAF (Figure 5D), suggesting that TPL2i does not compromise KRAS-RAF interaction and TPL2 activates MEK independently of RAFs. Apart from MAPK, TPL2i also dose-dependently suppressed p-p105, but this did not lead to increased p50 processing. Notably, TPL2i potently suppressed p-p65 at S276 in 3 out of 4 cell lines, but not S536 (Figure 5, D and E), consistent with published studies (30). Conversely, ectopic expression of TPL2 dosedependently increased p-p105, p-MEK, and p-ERK levels in 293T cells (Supplemental Figure 5A). These results show that TPL2 controls both MAPK and NF-KB cascades in PDAC.

Like the RAF kinases, TPL2 is a MAP3K that activates MEK. However, in RAS-mutant cells, BRAF inhibitors such as PLX-4720 and dabrafenib paradoxically hyperactivate MAPK cascades, which we also observed in KRAS-mutant HPAC, MIA Paca-2, and Pa01C cells expressing an SRE-driven luciferase reporter (Figure 5F and Supplemental Figure 5, B and C). In contrast, TPL2i dose-dependently suppressed SRE reporter activity in all 3 cell lines to levels matching those of MEK and ERK inhibitors. Notably, the MEK inhibitor trametinib unexpectedly enhanced NF- $\mathrm{KB}$ reporter activity in PDAC cells, perhaps representing a resistance mechanism underlying the lack of clinical efficacy of MEK inhibitors. On the other hand, TPL2i and IRAK4i dose-dependently suppressed both SRE and NF- $\mathrm{KB}$ reporter activities in KRAS-mutant PDAC cells (Supplemental Figure 5D). Notably, neither TPL2i nor IRAK4i suppressed p-MEK and p-ERK in 293T cells transfected with oncogenic BRAF ${ }^{V 600 E}$ (Supplemental Figure 5E), or in a BxPc-3 PDAC line that harbors an in-frame gain-of-function $B R A F$ mutation and WT KRAS (Supplemental Figure 5F), demonstrating IRAK4 and TPL2 as separate activators of MAPK, other than RAFs, downstream of KRAS.

Supporting an essential role of TPL2 in KRAS-induced transformation, TPL2i dose-dependently suppressed 3-dimensional (3D) AI and 2D clonogenic growth of HPNE-KRAS ${ }^{G 12 D}$ cells, HEKT/ tH-KRAS G12V cells, conventional PDAC lines, and PDCLs (Figure 5G and Supplemental Figure 6A). Knockdown of TPL2 by shRNA (shMAP3K8) severely impaired HPAC and Pa01C cell proliferation (Figure $5 \mathrm{H}$ ). The subpopulation that eventually grew again had only partial TPL2 knockdown but displayed reduced p-MEK, p-ERK, and p-p105 levels (Figure 5I) and were markedly impaired in 2D clonogenic growth (Figure 5J), and 3D growth as organoids or soft-agar colonies (Figure 5K and Supplemental Figure 6B). Together, these data establish a critical role for TPL2 in PDAC and KRAS signaling via supporting both NF-kB and MAPK pathways.

KRAS induces autocrine IL-1 $\beta$ inflammatory signaling to activate IRAK4 and TPL2. Next, we investigated the mechanism by which KRAS activates the IRAK4/TPL2 axis. IRAK4 and TPL2 are typically activated downstream of IL-1, TNF- $\alpha$, and TLRs, and not directly by KRAS. On this assumption, we surveyed the expression of IL- $1 \alpha / \beta$, TNF- $\alpha / \beta$, IL-1R, and all the TLRs by qRT-PCR of HEK $\mathrm{T} / \mathrm{tH}$ cells expressing an empty vector or KRAS ${ }^{\mathrm{G} 12 \mathrm{~V}}$. Of all 16 targets, only $I L 1 B$ mRNA was significantly upregulated (by $\sim 10$-fold) in $K R A S^{G 12 V}$-expressing cells compared with vector control (Figure 


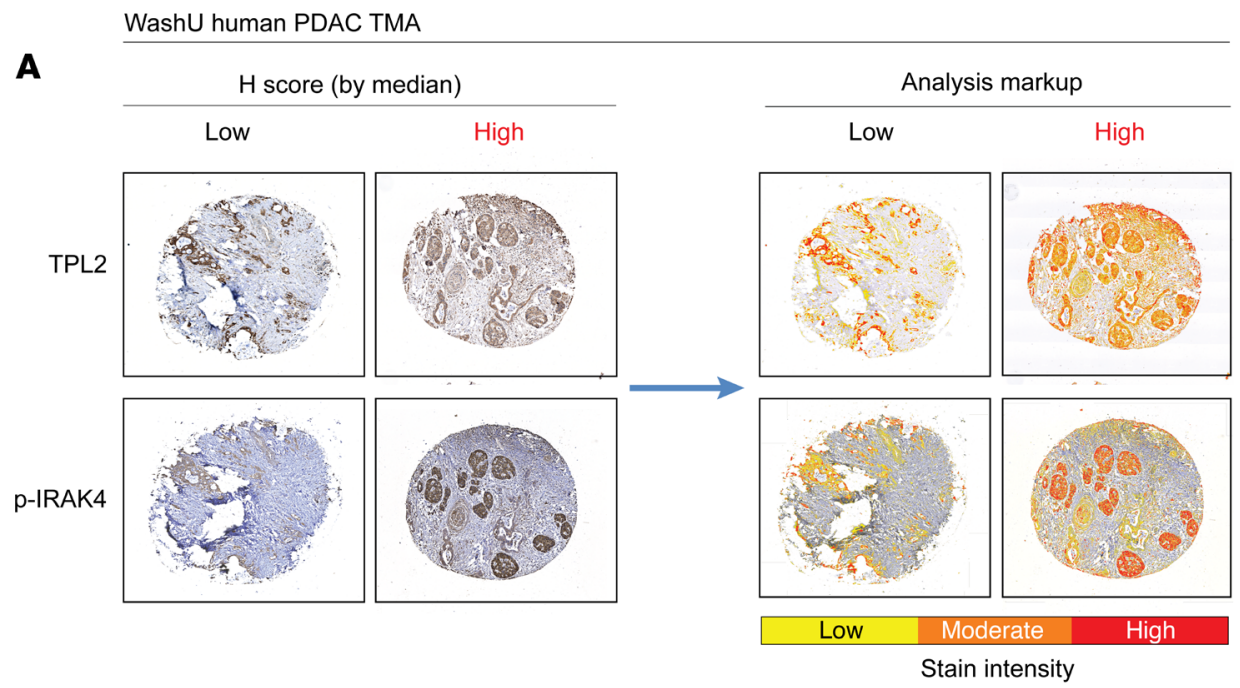

WashU human PDAC TMA

B

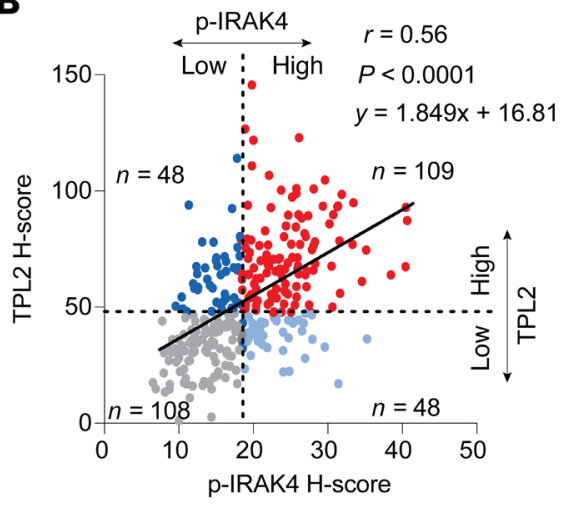

$\mathbf{E}$

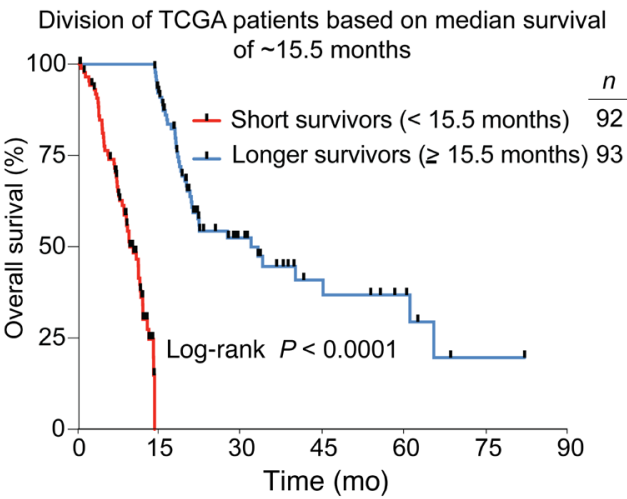

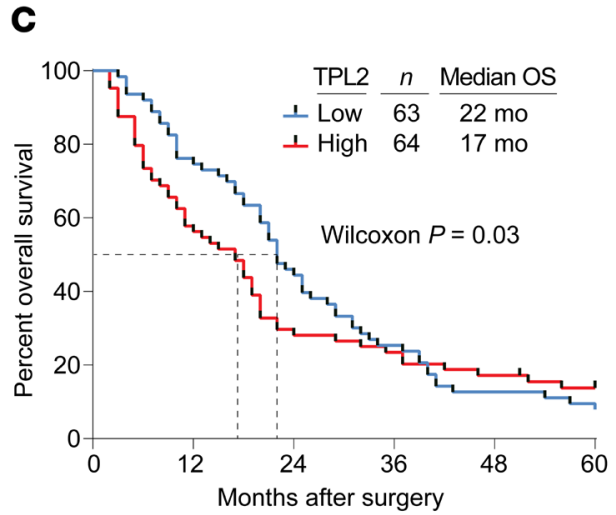

$\mathbf{F}$

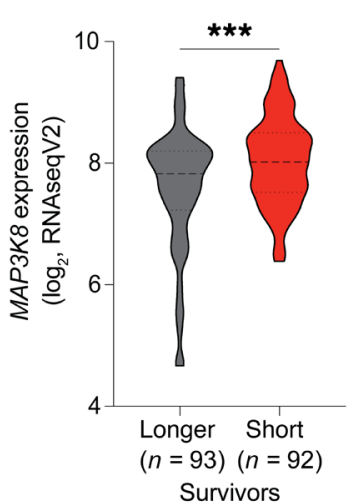

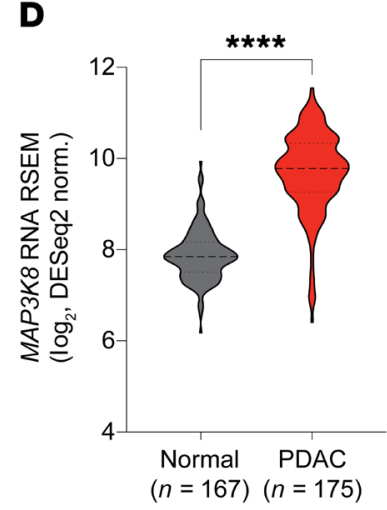

G

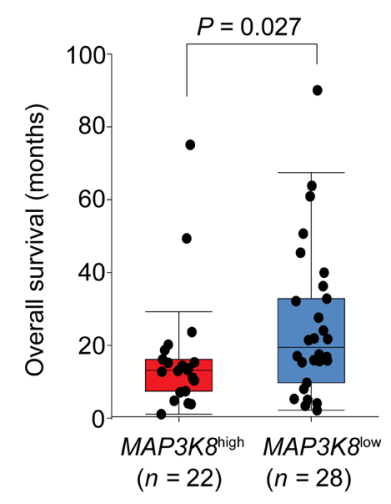

Figure 4. High TPL2 expression is poorly prognostic in PDAC. (A) IHC images representing high and low staining H-scores for TPL2 and p-IRAK4 with and without HALO analysis markup. H-score $=3 \times(\%$ of strongly stained area $)+2 \times(\%$ of moderately stained area $)+1 \times(\%$ of weakly stained area). (B) Spearman $(r)$ correlation plot of TPL2 and p-IRAK4 H-scores from tissue microarray (TMA) analysis of 313 tissue specimens from 157 PDAC patients, represented in A. (C) Kaplan-Meier plot comparing survival of patients with high vs. low TPL2 protein expression based on analysis of TMA above. Of the patients with survival data, those who died within 1 month of surgery were excluded from the analysis. (D) Graph depicting MAP3K8 (TPL2) expression in normal human pancreas versus PDAC tissue. Data for normal pancreas tissue is from the Genotype-Tissue Expression (GTEx) project and PDAC expression was from the pancreatic adenocarcinoma TCGA PanCancer Atlas study. $P$ values are from unpaired, 2 -sided $t$ test. Outliers (5 in normal, 3 in PDAC) were removed by ROUT, $\underline{Q}=0.1 \%$. (E) Graph of overall survival (OS) of TCCA PDAC patients separated into short- and longer-surviving cohorts using median OS of approximately 15.5 months. (F) Graph comparing MAP3K8 mRNA expression in longer- vs. short-surviving patients defined in $\mathbf{E}$. $P$ values are from unpaired, 2-sided $t$ test. All 178 (out of 185) TCCA PDAC samples with mRNA expression data were analyzed. (C) Graph comparing months of OS of patients with high ( $Z$ score $>1, n=22$ ) versus low ( $Z$ score $<1, n=28$ ) MAP3K8 expression based on analysis of TCGA Firehose Legacy data set. $P$ values from Kruskal-Wallis test. ${ }^{* * *} P<0.0001 ;{ }^{* * *} P<0.0002$. 
6A), which we confirmed by IL-1 $1 \beta$ ELISA of conditioned media (CM) collected from these cells ( 120-fold higher in KRASG12V expressing cells, Supplemental Figure 7A). Furthermore, analysis of oncogenic KRAS signature showed IL1B expression was significantly higher in $M A P 3 K 8^{\text {High }}$ patients (Figure 6B). These results suggest autocrine IL-1 $\beta$ is the driver of IRAK 4 and TPL 2 activity in $K R A S$-mutant cells. In support, KRAS ${ }^{G 12 V}$ expression upregulated p-IRAK4, p-TPL2, p-MEK, and p-ERK levels in HEK T/tH cells, but all were dose-dependently reversed by treatment with a neutralizing anti-human IL-1 $\beta$ (anti-hIL-1 $\beta$ ) antibody (Figure 6C). CM collected from HEK-T/tH KRAS ${ }^{G 12 V}$ cells was able to upregulate p-TPL2, p-IRAK4, p-MEK, and p-ERK levels in HEK T/tH cells, but the effect was blunted with neutralizing anti-hIL-1 $\beta$ antibody (Figure 6D), shRNA knockdown of IL-1R of recipient HEK T/tH cells (Supplemental Figure 7, B and C), and completely blocked by cotreatment with TPL2i (Figure 6E). In support of these data, CM from HEK-T/tH KRAS ${ }^{G 12 V}$ cells stimulated SRE reporter activity of HPAC cells by approximately 3-fold (Supplemental Figure 7D) and treatment of $\mathrm{HEK} \mathrm{T} / \mathrm{tH}, \mathrm{HPAC}$, and $\mathrm{PaO1C}$ cells with recombinant hIL-1 $\beta$ upregulated p-IRAK4, p-TPL2, p-MEK, p-ERK, and p-RSK (Figure 6F and Supplemental Figure 7E). Knockdown of IL-1R also blunted upregulation of p-MEK and p-ERK in HEK-T/tH transfected with $K R A S^{G 12 V}$ or MAP3K8 (TPL2) (Figure 6G), with similar effects observed in KRAS-mutant Pa01C cells (Supplemental Figure 7F). These data establish autocrine IL-1 $\beta /$ IRAK4/TPL2 signaling as a parallel mechanism, apart from RAF kinases, through which KRAS oncoprotein drives MAPK signaling.

We next investigated the mechanism by which KRAS promotes IL-1 $\beta$ production. We treated HEK T/tH KRAS ${ }^{G 12 V}$ cells with MEK (trametinib), ERK (ulixertinib), or PI3K (GDC-0941) inhibitors and found that both MEK and ERK inhibitors significantly abrogated IL-1 $\beta$ production, whereas the PI3Ki had no effect (Supplemental Figure 7G). These data depict a model in which KRAS oncoprotein uses the MAPK effectors to promote IL-1 $\beta$ production and create an autocrine IL-1 $\beta$-IL-1R-IRAK4-TPL2-MAPK feedforward loop that amplifies MAPK signaling. From TCGA database, elevated $I L 1 B$ expression associated with poor prognosis in PDAC (Figure 6H). The mRNA level of TPL2 (MAP3K8) moderately $(r=0.36)$ and strongly $(r=0.5)$ positively correlated with $I L 1 B$ and IL1R1, respectively (Supplemental Figure 8, A and B). In KP2 cells, CRISPR knockout of Irak4 led to downregulation of GO signatures associated with IL-1, and reexpression of Irak4 (rescue) significantly restored these signatures (Supplemental Figure 8, C and D). In these gene sets, TPL2 was present in the leading edge as a core enrichment in an IRAK4-dependent manner (Supplemental Figure 8E), again signifying its role in IL-1 and cytokine signaling. Together, these data establish autocrine IL-1R/IRAK4/TPL2 signaling as an essential mechanism hijacked by KRAS that should be therapeutically targeted.

TPL2 inhibition potentiates chemotherapy by curbing MAPK and $N F-\kappa B$ activation. Molecularly targeted therapies have been ineffective in treating PDAC patients. Therefore, it is unlikely that TPL2 inhibition or IRAK4 inhibition alone will be clinically effective, and combination regimens will need to be developed. Chemotherapy is currently the only effective treatment modality for PDAC, but treatment response is neither universal nor durable $(31,32)$. Stress-induced NF- $\mathrm{B}$ and MAPK survival signaling are among the multiple mechanisms that underlie de novo chemoresistance. To address this, we examined whether the IRAK4/ TPL2 axis contributes to chemotherapy-induced survival and resistance, which will help formulate a rational combinatorial regimen for in vivo testing. We treated PDAC cells with 5 chemotherapeutic agents (gemcitabine, paclitaxel, SN-38, 5-fluorouracil [5-FU], and oxaliplatin) commonly used in patient care. Of these agents, SN-38, an active metabolite of irinotecan, was the most potent in inducing $\mathrm{p}$-ERK, p-MEK, p-RSK, and notably p-TPL2 and p-IRAK4, across multiple PDAC lines (Figure 7A and Supplemental Figure 9A), suggesting IRAK4 and TPL2 may contribute to MAPK activation following genotoxic stress. NF- $\kappa \mathrm{B}$ was also induced, as evident by an increase in p-p105.

To determine the mechanism that activates these markers, we treated HPAC cells with gemcitabine/paclitaxel or FIRINOX (5-FU/SN-38/oxaliplatin), which mimic clinical regimens, and surveyed changes in expression of the TLRs and IL- $1 \alpha / \beta$ in HPAC cells. Intriguingly, we observed significantly upregulated expression of TLR6, TLR9, and IL1A, but not $I L 1 B$, upon exposure to either chemotherapy (Figure 7B). Survey of 2 other PDCLs, $\mathrm{Pa} 01 \mathrm{C}$ and $\mathrm{PaO3C}$, showed TLR9 to be the only gene consistently upregulated following FIRINOX treatment (Figure 7C). Importantly, signaling from TLR9 is transmitted exclusively through IRAK4 (33). Indeed, proximity ligation assay showed markedly increased TLR9 and p-IRAK4 interaction following treatment of 3 different PDAC lines with SN-38 (Figure 7D), suggesting TLR9 to be the driver of IRAK4 and TPL2 upon chemotherapy exposure. Interestingly, analysis of TCGA data also revealed a strong and significant correlation (Pearson $r=0.49, P=2.61 \times 10^{-12}$ ) between TLR9 and MAP3K8 (TPL2) mRNA expression in PDAC samples (Supplemental Figure 9B). Cotreatment with TPL2i significantly attenuated SN-38-induced p-MEK, p-ERK, and p-RSK, and increased poly(ADP-ribose) polymerase (PARP) cleavage in PDAC lines (Figure 7E). Gemcitabine-induced p-MEK and p-ERK levels and SRE activity were also suppressible with TPL2i (Supplemental Figure 9, C and D), suggesting that induction of MAPK activity is not specific to one class of cytotoxic agent. Besides the MAPK cascade, the TPL2i additionally suppressed p-p105 in SN-38-treated PaO1C cells (Supplemental Figure 9E), making it a more broad-spectrum therapeutic agent than the MEK inhibitor or the ERK inhibitor in curbing chemotherapy-induced survival signaling. PDAC cells cotreated with SN38 and TPL2i showed significantly more apoptosis (Supplemental Figure 9, F and G) by flow cytometric analysis, and completely lost their clonogenicity, an assay that tests emergence of resistant clones (Figure $7 \mathrm{~F}$ and Supplemental Figure 10A). Notably, the combination of TPL2i and SN-38 was synergistic in suppressing PDAC cell growth by the Loewe additivity model (Supplemental Figure 10B). Accordingly, the combination of TPL2 $i$ and FIRINOX was significantly more efficacious in suppressing in vivo subcutaneous growth of $\mathrm{PaO1C}$ tumors compared with either treatment alone (Figure 7G and Supplemental Figure 10, C and D), and without incurring noticeable toxicities (Supplemental Figure 10E). TPL2i alone was effective in significantly suppressing growth of orthotopic KI tumors in syngeneic FVB/NJ mice. When combined with FIRINOX, the mean tumor burden was seemingly further reduced, with 2 mice showing no evidence of cancer, although the difference between TPL2i 
A

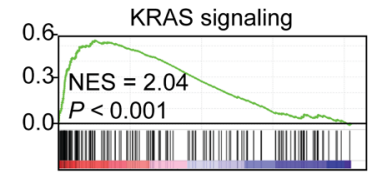

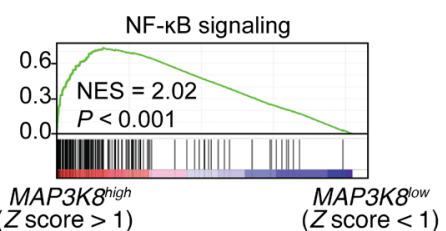

B

Pa01C Pa02C Pa03C Pa14C PANC-1 HPAC TPL2i $(\mu M): \overline{V 510} \overline{V 510} \overline{\text { V } 510} \overline{\text { V } 510} \overline{\text { V } 510} \overline{\text { V } 510}$

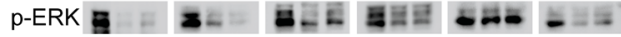

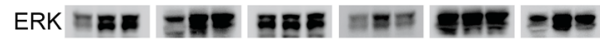

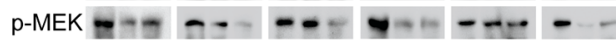

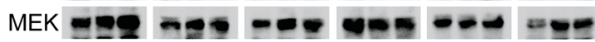
GAPDH - - - - - - - - - -
C

293T

293T KRAS $12 \mathrm{~V}$

TPL2i $(\mu M): \vee 510 \vee 510$ p-ERK $\quad \approx=$

ERK $=\mathbf{x = m a}$

p-MEK - -

MEK -

GAPDH ローーாロ
D

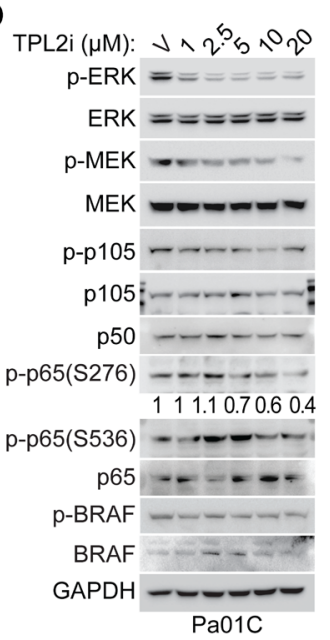

E $\stackrel{\text { HPAC }}{\mathrm{PaO02C}} \stackrel{\mathrm{Pa03C}}{V \mathrm{~T}}$ TPL2i $(\mu M): V 510 \vee 510 \vee 510$ p-p105 - - - p105 - - .

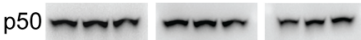
p-p65 (S276) - - - - - -

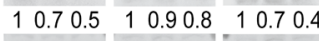
p-p65 (S536) ーละ -ோ -

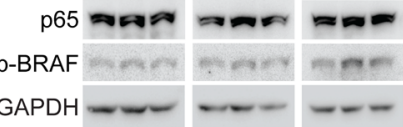

$\mathbf{F}$

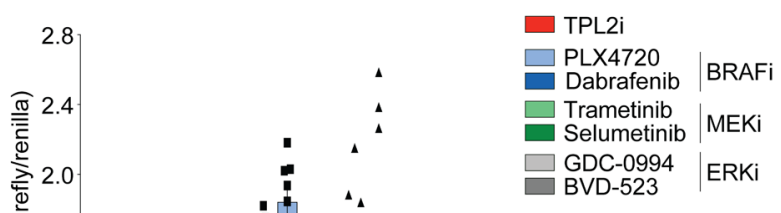

G TPL2i

G $\square$ Vehicle $\square .5 \mu \mathrm{M}$

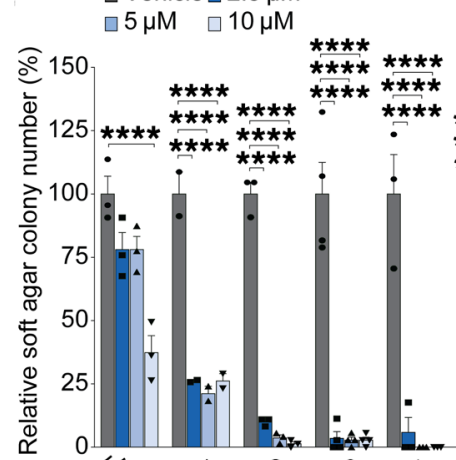

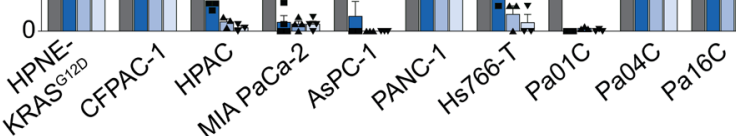

H

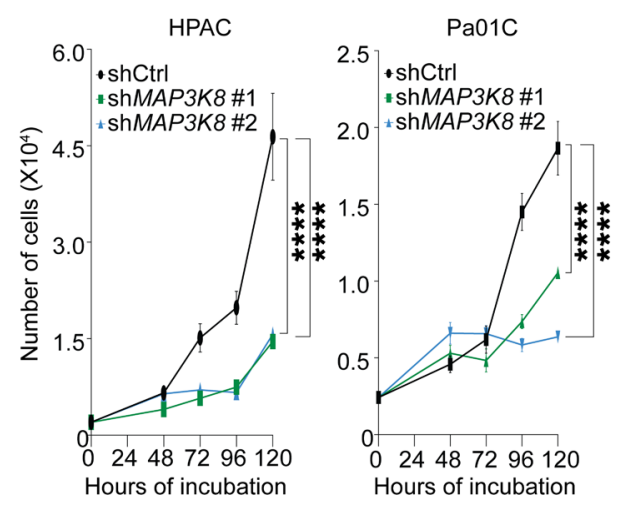

I

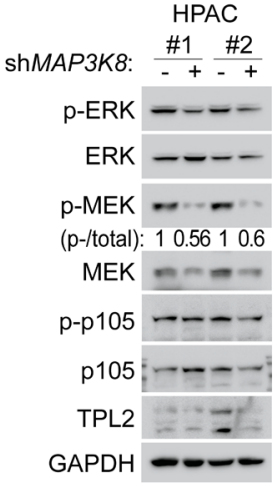

J

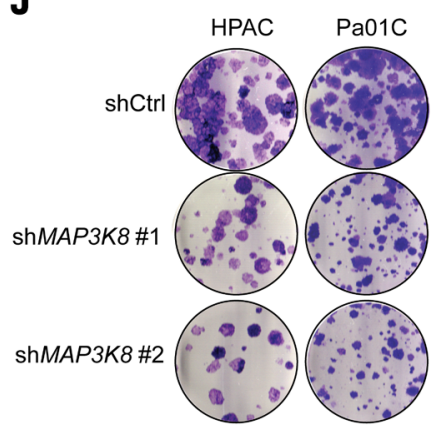

$\mathbf{K}$

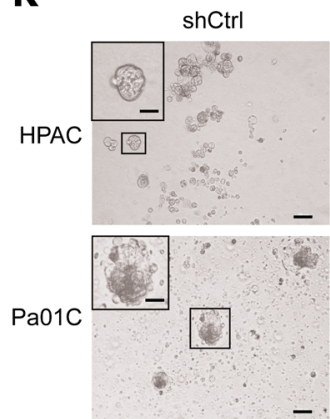

ShMAP $3 K 8 \# 2$

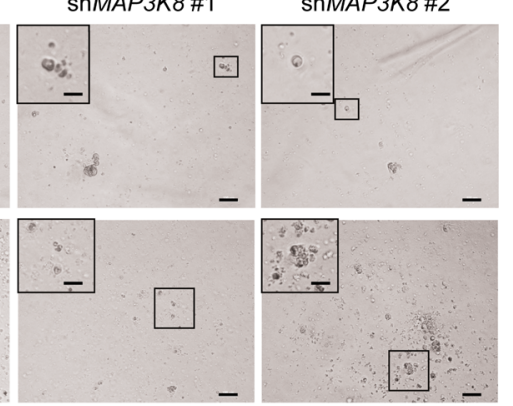

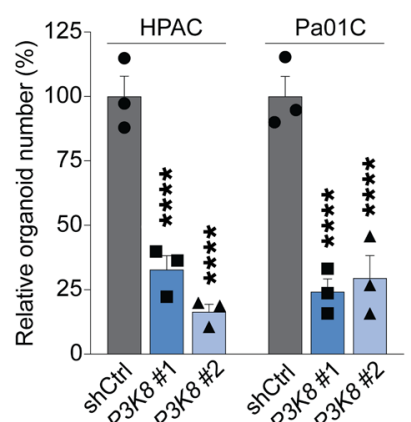


Figure 5. TPL2 drives both MAPK and NF- $\mathrm{KB}$ signaling in PDAC. (A) Gene set enrichment plots for patients with high ( $Z$ score $>1, n=22$ ) and low ( $Z$ score $<1, n=28$ ) MAP3K8 (TPL2) expression from TCGA Firehose Legacy study. (B) Immunoblots of KRAS-mutant human PDAC cell lines treated with TPL2 inhibitor (TPL2i) or vehicle (V) for 36 hours in serum-free media. (C) Immunoblots of 293T and 293T-KRASG12V cells treated with TPL2i for 24 hours in serum-free condition. (D) Immunoblots of Pa01C cells treated with incremental doses of TPL2i. (E) Immunoblots of PDAC cell lines treated with TPL2i. (F) Serum-response element (SRE) reporter assay of HPAC cells treated with TPL2i, BRAFi, MEKi, or ERKi. Data show 3 independent experiments, each done with triplicate samples. (C) Quantification of softagar colonies formed by PDAC cell lines treated with TPL2i. Data represent $n=3$ ( $n=2$ for CFPAC-1) for each cell line. One data point is shown per biological replicate, each consisting of 3 technical replicates. $P$ values from 2-way ANOVA with Dunnett's multiple-comparisons test. (H) Immunoblots of WT or MAP3K8-knockdown HPAC cells. (I) Quantification of HPAC and Pa01C cell proliferation after TPL2 knockdown with shRNA (shMAP3K8). Each data point is the average of 6 replicates. $P$ values by 2-way ANOVA with Dunnett's multiple-comparisons test. (J) Representative crystal violet-stained images of $2 \mathrm{D}$ colony formation of TPL2-knockdown HPAC and PaO1C cells. (K) Light microscopic images of organoids formed by HPAC and Pa01C cells with TPL2 knockdown. Scale bars: $100 \mu \mathrm{m}$ (full images) and $25 \mu \mathrm{m}$ (insets). Graph on right is quantification of number of organoids formed in 3 independent wells. $P$ values by 2-way ANOVA with Dunnett's multiple-comparisons test. All data presented as mean \pm SEM. ${ }^{* * *} P<$ $0.0001 ;{ }^{* *} P<0.0002 ;{ }^{*} P<0.0332$.

and combo arms did not reach statistical significance probably due to low mouse number per arm (Figure $7 \mathrm{H}$ ). These results show that targeting TPL2 overcomes genotoxic stress-induced survival signaling and enhances the efficacy of chemotherapy.

MAP3K8 point mutations, E188K and $\mathrm{R} 442 \mathrm{H}$, hyperactivate $M A P K$ and $N F-\kappa B$ cascades. Aside from being summoned by oncogenic RAS and genotoxic stress, TPL2 is also spontaneously activated by genetic mutations. In the basal state, TPL2 protein is bound and inhibited by p105 (NF- $\mathrm{BB} 1)$ and A20-binding inhibitor of NF- $\kappa \mathrm{B}$ (ABIN-2). Activated IKK complex phosphorylates p105 and prompts its proteolysis to p50, which releases TPL2 (34). TPL2 undergoes phosphorylation at residues S400 by IKK and T290 by an unknown kinase to become fully activated, after which it is proteasomally degraded via polyubiquitination at its C-terminus (35-37). Therefore, C-terminally truncated TPL2 is more stable and potent in activating the MAPK pathway (38). Oncogenic truncations and fusions of $M A P 3 K 8$ are reported in spitzoid melanomas and predict sensitivity to MEK inhibitors in vitro (39). Besides truncations, from TCGA database we noticed several point mutations in MAP3K8 across various non-PDAC cancer types that have not been characterized. Therefore, we investigated 5 point mutants that occur with the highest frequency: E188K, R397H, R442H, L444V, and R459W (Figure 8A). Of these mutants, we found $\mathrm{E} 188 \mathrm{~K}$ and $\mathrm{R} 442 \mathrm{H}$ to be the most potent in stimulating SRE and $\mathrm{NF}-\kappa \mathrm{B}$ reporter activities (Supplemental Figure 11, A and B), which we characterized in further detail. The $\mathrm{E} 188 \mathrm{~K}$ mutation is detected in oligodendroglioma and colon and urothelial carcinoma, whereas $\mathrm{R} 442$ can acquire missense (to $\mathrm{H}$ or $\mathrm{C}$ ) or nonsense mutations in colon, ovarian, and gastric cancers, and rhabdoid tumors.

When expressed at the same level as WT, TPL2 $2^{\mathrm{E} 188 \mathrm{~K}}$ is markedly more potent in activating MEK, ERK, RSK, and p105 (Figure $8 \mathrm{~B}$ ), as well as inducing SRE and $\mathrm{NF}-\mathrm{\kappa B}$ reporter activities (Figure $8 \mathrm{C}$ ) in unperturbed $293 \mathrm{~T}$ cells, clearly showing this is a gain-of- function mutation. To comprehensively evaluate the impact of the E188K mutation, we performed RPPA analysis on 293T cells ectopically expressing vector, and a roughly equal amount of TPL $2^{\text {WT }}$ or TPL2 ${ }^{\mathrm{E} 188 \mathrm{~K}}$ (Figure $8 \mathrm{~B}$ ). Of all 441 markers analyzed, p-ERK is the most differentially upregulated marker in TPL $2^{\mathrm{E} 188 \mathrm{~K}}$-expressing cells (Figure 8D), validating our findings with immunoblots and reporter assay. We also observed significantly increased p-S6, a known substrate of ERK, and p-c-Jun, which has been described as a TPL2 effector (ref. 30 and Figure 8E). This analysis showed, in a comprehensive and unbiased manner, the MEK/ERK cascade to be the predominant signaling cascade activated by the E188K mutation.

To delineate the molecular mechanism underlying the enhanced kinase activity of TPL $2^{\mathrm{E} 188 \mathrm{~K}}$, we first compared the halflives of TPL2 ${ }^{\mathrm{WT}}$ and TPL2 ${ }^{\mathrm{E} 188 \mathrm{~K}}$ in $293 \mathrm{~T}$ cells treated with a protein synthesis inhibitor, cycloheximide. Surprisingly, we found that the E188K mutation, despite being located within the kinase domain, rendered the TPL2 protein more stable (Figure 9A). Supporting this finding, immunoprecipitation assay showed that the E188K mutation almost completely abolished TPL2 polyubiquitination, a prerequisite for proteasomal degradation (Figure 9B). To evaluate the oncogenicity of TPL2 ${ }^{\mathrm{E} 188 \mathrm{~K}}$, we obtained Hs695T, a $B R A F^{V 600 E}$-mutant melanoma cell line that naturally harbors the $M A P 3 K 8^{E 188 K}$ mutation. Sanger sequencing of cDNA from Hs695T confirmed a $\mathrm{G} \rightarrow \mathrm{A}$ mutation in codon 188 of $M A P 3 \mathrm{~K} 8$, which converts glutamate (GAG) to lysine (AAG) (Figure 9C). We therefore speculate that TPL2 $2^{\mathrm{E} 188 \mathrm{~K}}$ may be an oncoprotein driving MAPK activation in this cell line. Supporting this notion, treatment with TPL2i suppressed p-ERK and p-MEK (Figure 9D) as well as in vitro proliferation and viability of Hs695T cells (Figure 9E and Supplemental Figure 11C). Importantly, the BRAF inhibitor PLX4032 had a modest growth suppressive effect, but when combined with TPL2i, it significantly lowered $(P<0.0001)$ the viability of Hs695T (Figure 9F), indicating that both BRAF and MAP3K8 mutations contribute to MAPK activity in this cell line.

We next determined the effect of mutation at codon 442 , which resides in the C-terminus, implying disruption of degradation function. We chose to study the $\mathrm{R} 442 \mathrm{H}$ mutant because it is the most common mutation described in TCGA and more potent in inducing SRE and NF- $\kappa \mathrm{B}$ reporter activity than WT protein (Supplemental Figure 11, A and B). Compared with TPL2 ${ }^{\mathrm{WT}}$, TPL $2^{\mathrm{R} 442 \mathrm{H}}$ is more potent in activating MEK, ERK, and RSK (Figure 10A) when expressed at equivalent levels. As expected, the stability of TPL $2^{\mathrm{R} 442 \mathrm{H}}$ mutant protein was higher than TPL $2^{\mathrm{WT}}$ and comparable to C-terminally truncated TPL2 (Figure 10B). Interestingly, other mutations including $\mathrm{L} 444 \mathrm{~V}$ and $\mathrm{R} 459 \mathrm{~W}$ also render TPL2 slightly more stable than the WT form, albeit not to the degree imparted by $\mathrm{R} 442 \mathrm{H}$. All these mutations reduce polyubiquitination of TPL2 (Figure 10C), which explains their increased stability. The naturally occurring $M A P 3 K 8^{R 442 H}$ mutation has been reported in the ovarian cancer cell line IGROV1 (40). Remarkably, short-duration (2 hours) treatment with a very low dose of TPL2i $(1 \mu \mathrm{M})$ completely blocked p-MEK, p-ERK, and p-RSK in this cell line to undetectable levels even with high exposure time (Figure 10D), suggesting that TPL2 is the dominant driver of MAPK activity in this cell line. In support of this, partial knockdown of TPL2 also reduced MAPK activity in IGROV1 (Figure 10E). Pharmacologic inhibition or silencing of TPL2 significantly 
A

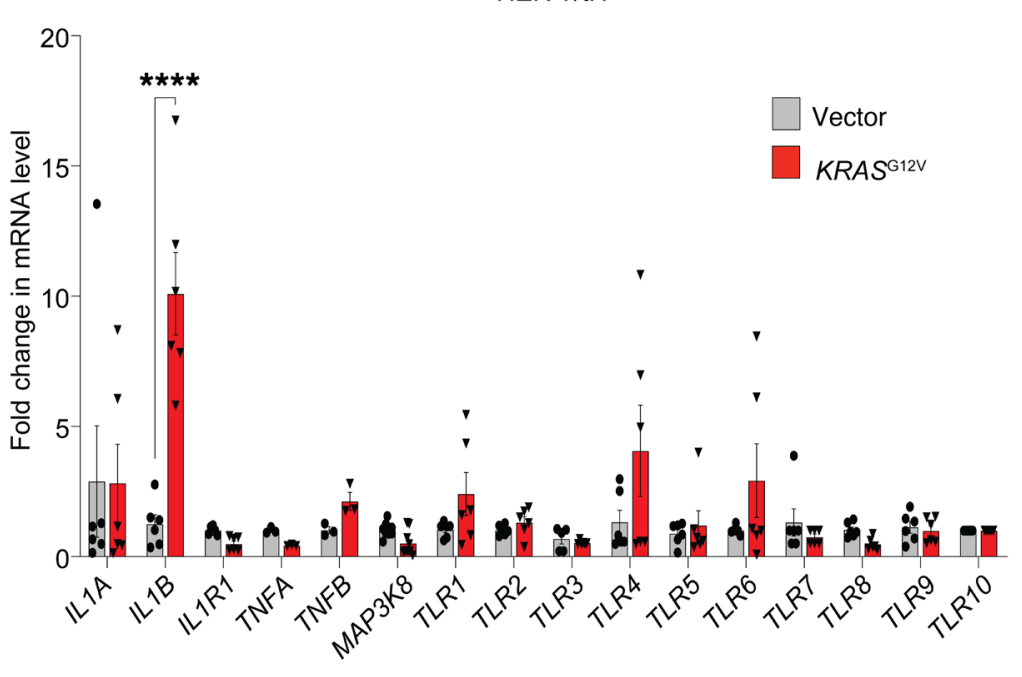

B
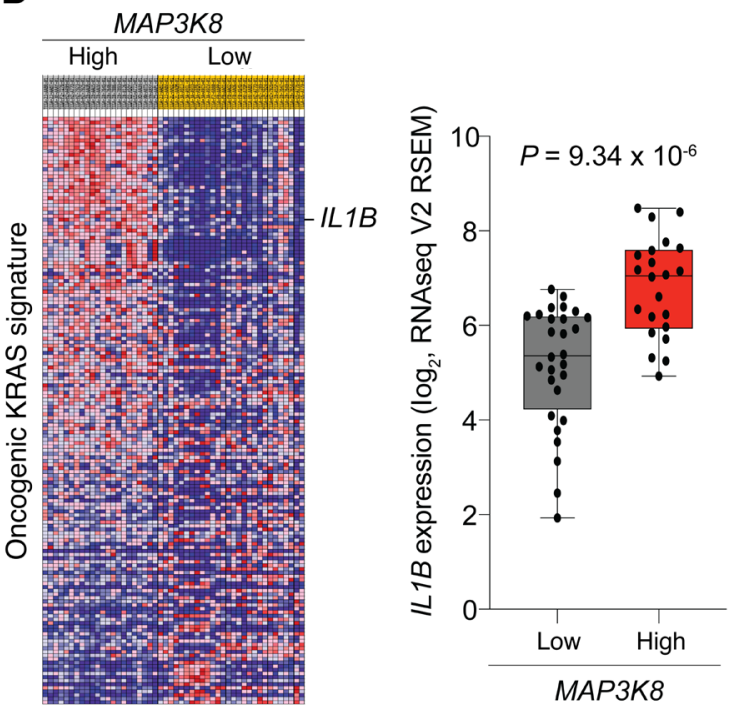

C

D

HEK T/LH

EV KRAS ${ }^{\mathrm{G} 2 \mathrm{~V}}$ $\alpha$-hIL-1 $\beta(\mu \mathrm{g} / \mathrm{mL}): 0024$ p-ERK - - -

$\mathrm{ERK}=--$

p-MEK - - -

MEK $=$ -

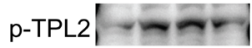

TPL2 $\approx= \pm \forall$

p-IRAK4 -....

IRAK4' -

p-p105 …n.

p105:-...

p50 ーேーニー

GAPDH

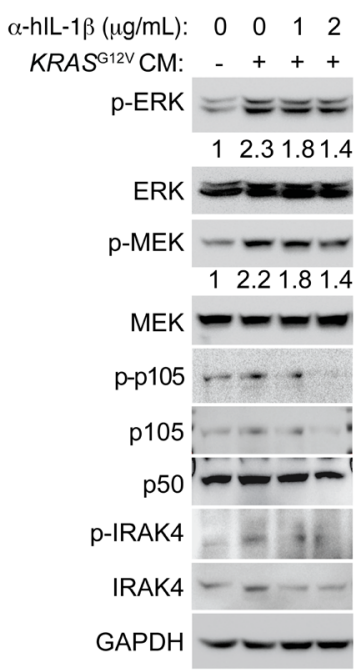

$\alpha-h \mid l-1 \beta(\mu \mathrm{g} / \mathrm{mL}): \quad \begin{array}{llll}0 & 0 & 1 & 2\end{array}$ P-ERK

12.31 .81 .4

G

HPAC

EV HA-TPL2 WT EV HA-TPL2 WT hlL-1 $\beta$ (mins): $0 \quad 05101520 \quad 0 \quad 05101520$

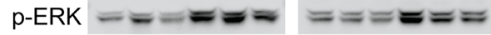

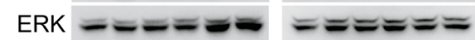

p-MEK - - -

MEK =யா=யா -ாயாய

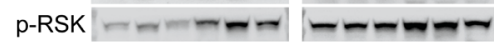

RSK 물 -

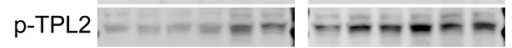

HA-TPL2 -

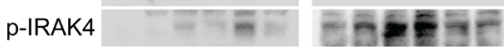

IRAK4:-

GAPDH - -
E

KRAS ${ }^{\mathrm{G} 12 \mathrm{~V}} \mathrm{CM}:-++$

$\operatorname{TPL} 2 \mathrm{i}(\mu \mathrm{M}): \quad \vee \quad \vee 510$

p-ERK $==-$

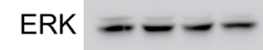

p-MEK - -

MEK - -

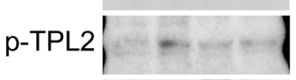

TPL2

GAPDH

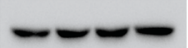

HEK T/tH

HA-TPL2 WT: $\quad-\quad++++$ KRASG12V: $-\quad+++$ shIL1R1: - - - \#1 \#2 p-ERK $--=-$ ERK - - = a p-MEK $=\cdots=$ MEK GAPDH
H

IL1B

(PDAC, TCGA provisional)

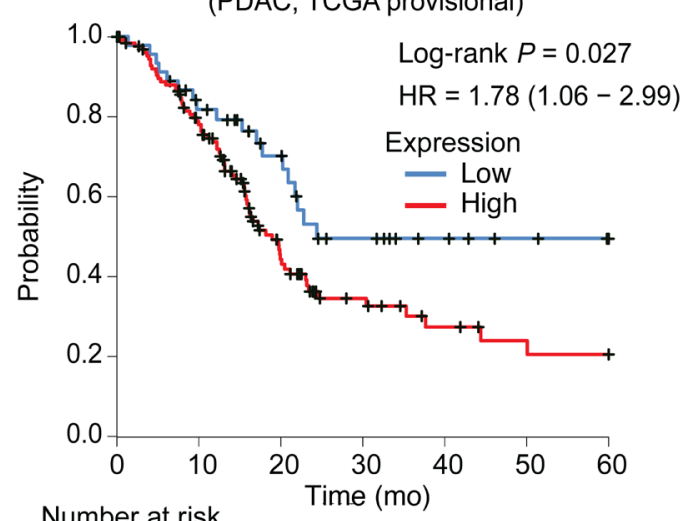

Number at risk

$\begin{array}{llllcll}\text { Low } 46 & 33 & 22 & 12 & 7 & 4 & 2 \\ \text { High } 131 & 91 & 36 & 18 & 10 & 7 & 6\end{array}$ 
Figure 6. KRAS induces autocrine IL-1 $\beta$ signaling, which activates IRAK4 and TPL2. (A) qRT-PCR of HEK cells expressing empty vector or KRASG12V. Data show 6 replicates from 2 independent experiments. ${ }^{* * *} P<0.0001$ by 2-way ANOVA with Dunnett's multiple-comparisons test. (B) Heatmap of Hallmark "KRAS signaling up" signature in MAP3K8-high vs. -low patients. IL1B is significantly enriched in MAP3K ${ }^{\text {High }}$ patients, shown also in box-and-whisker plot on right. Significance tested with unpaired, 2 -sided $t$ test. (C) Immunoblots of HEK-KRAS ${ }^{12 V}$ cells treated with antihIL-1 $\beta$ neutralizing antibody for 24 hours. (D) Immunoblots of HEK cells incubated with conditioned media (CM) from HEK-KRASG12V cells (called "KRASG12V (M") and anti-hIL-1 $\beta$ neutralizing antibody. (E) Immunoblots of HEK cells incubated with KRASC12V CM and TPL2i or vehicle (V) for 16 hours. (F) Immunoblots of HEK and HPAC cells overexpressing HA epitopetagged WT TPL2 stimulated with $100 \mathrm{ng} / \mathrm{mL}$ recombinant hIL-1 $\beta$ for the indicated duration. (G) Immunoblots of HEK cells expressing KRASG12V, HA epitope-tagged WT TPL2, and shIL1R1. (H) Kaplan-Meier curve of PDAC TCGA patients with high vs. low IL1B expression. Follow-up censored at 60 months. All data presented as mean \pm SEM.

decreased the proliferation, colony forming ability, and AI growth of IGROV1 (Figure 10, F and G, and Supplemental Figure $12, \mathrm{~A}-\mathrm{C})$, consistent with prior reports (41). Importantly, while knockdown of TPL2 markedly crippled the ability of IGROV1 to grow into $3 \mathrm{D}$ organoids or 2D clones, these growths could only be rescued by reexpression of TPL $2^{\mathrm{R} 442 \mathrm{H}}$ but not TPL $2^{\mathrm{WT}}$ (Figure $10 \mathrm{H}$ and Supplemental Figure 12D). Overall, these studies are the first to our knowledge to describe gain-of-function point mutations of MAP3K8 (TPL2) in human cancers, further prompting the need to identify these mutations in clinical practice and develop dedicated TPL2 inhibitors for clinical trials.

\section{Discussion}

Our present study provides broader understanding of the role of IRAK4 and TPL2 in human cancers. Using genetically defined cell lines and PDAC as a disease model, we show that KRAS oncoprotein uses the MAPK cascade to upregulate IL- $1 \beta$ production, leading to autocrine activation of IRAK4 and TPL2, which feeds back to escalate MAPK activity and additionally the NF-KB cascade. Following genotoxic stress, PDAC cells upregulate TLR9, leading to enhanced utilization of the IRAK4/TPL2 axis to sustain survival. Additionally, we characterize what we believe to be novel gain-of-function TPL2 mutations that hyperactivate the MAPK and NF- $\mathrm{KB}$ pathways.

The malignant feats of RAS oncoproteins result from direct and indirect signaling mechanisms. RAS oncoproteins can directly bind and activate several effectors including the RAF kinases, PI3 kinases, RalGEFs, and Tiam1 (2). Through these pathways, a plethora of inflammatory chemokines and cytokines including IL-6, IL-8, IL-1 $\alpha / \beta$, and CCL5 are produced, which in an autocrine manner trigger the inflammatory JAK/STAT and NF-KB cascades $(10,22,42-46)$. These secondary events not only help propagate tumor progression, but also shield cancer cells from therapeutic attacks. For instance, in KRAS-mutant lung cancer, TBK1- and IKK-driven CCL5 and IL-6 can activate the JAK/ STAT pathway to confer resistance to MEK inhibitors (42). In PDAC, STAT3 activation drives resistance to MEK inhibitors $(44,45)$. Therefore, autocrine/paracrine cytokine signaling provides equally essential support, in addition to the intrinsic oncogenic events, that help cancer cells adapt and withstand stress.
In this study, we show the autocrine IL-1 $\beta$-driven IRAK4/TPL2 axis to be an essential component of KRAS and MAPK signaling. Because IRAK4 is typically activated by inflammatory receptors IL-1R, TLRs, and TNFR, it is most widely studied as the driver of NF- $\mathrm{KB}$ activity in immune cells (47). Strikingly, ablation of IRAK4 completely blocked RAS-induced transformation and tumorigenicity in both epithelial cells and fibroblasts, as well as PDAC cells. These data are in strong agreement with an elegant study by Ling et al. showing that ablation of IKK $\beta$, a key downstream substrate of IRAK4, completely abolished PDAC development in a KRAS/Ink4a mouse model (8). The IL-1R antagonist Anakinra is currently being tested in clinical trials in combination with chemotherapy for PDAC (NCT02550327). Downstream of IRAK4, we revealed TPL2 as a MAP3K that drives MEK/ERK and NF-kB p105 in KRAS-mutant cells independently of the RAF kinases. Therefore, TPL2 is a promising therapeutic target that controls multiple signaling cascades in KRAS-driven cancers.

Aside from enforcing KRAS autocrine signaling, the IRAK4/ TPL2 axis is further used following genotoxic stress as a survival mechanism. Induction of MAPK activity is a well-established mechanism that allows cancer cells to endure genotoxic stress (48). However, MEK inhibitors fail to potentiate chemotherapy in pancreatic cancer $(49,50)$, suggesting that targeting MAPK alone is insufficient, or that compensatory escape mechanisms such as enhanced NF- $\mathrm{kB}$ activity should be cotargeted. Similarly, in a prostate cancer model, addition of an NF-KB pathway inhibitor significantly potentiates the antitumor effect of MEK inhibitors (51). Here in our study, we found that PDAC cells adapt to chemotherapy by upregulating TLR9, which signals through IRAK4/ IKK/TPL2 to activate multiple pathways. Therefore, PDAC cells use different receptors, IL-1R at baseline and TLR9 during genotoxic stress, to engage the same IRAK4/IKK/TPL2 axis for survival. It is important to keep in mind that since the therapeutic spectrum of TPL2i extends beyond MEK/ERK, encompassing the NF- $\mathrm{\kappa B}$, JNK, and p38 cascades, all of which have been implicated in chemoresistance, TPL2 may be a more promising therapeutic target than MEK or ERK when combined with chemotherapy, at least in PDAC. In accordance, pharmacologic TPL2 inhibition completely blocked chemotherapy-induced MAPK and NF- $\mathrm{kB}$ activation, resulting in greater apoptosis and tumor suppression in vivo (Supplemental Figures 9, 10, and 13).

To the best of our knowledge, our study is the first to report gainof-function point mutations of TPL2. Overexpression, C-terminal truncations, or fusions of TPL2 have been found in T cell neoplasms, melanoma, ovarian, breast, and lung cancers. These mutations are associated with RAF inhibitor resistance, and can be targeted with MEK inhibitors (39, 41, 52-55). Compared with WT protein, the E188K and R442H mutants we studied are more stable and capable of hyperactivating both MAPK and NF- $\mathrm{kB}$ cascades. Furthermore, cancer cell lines naturally harboring these mutations (Hs695T for E188K, and IGROV1 for R442H) are highly sensitive to TPL2 inhibition. Importantly, TPL2i significantly suppressed MAPK activity and proliferation of $B R A F^{V 600 E} / M A P 3 K 8^{E 188 K}$ double-mutant Hs695T cells, but not in $B R A F^{V 600 E} / M A P 3 K 8^{W T}$ BxPc-3 cells, demonstrating that MAP3K $8^{E 188 K}$ is oncogenic.

The crystal structure of the C-terminus of TPL2 has not been resolved, and therefore how these mutations conformationally 
A

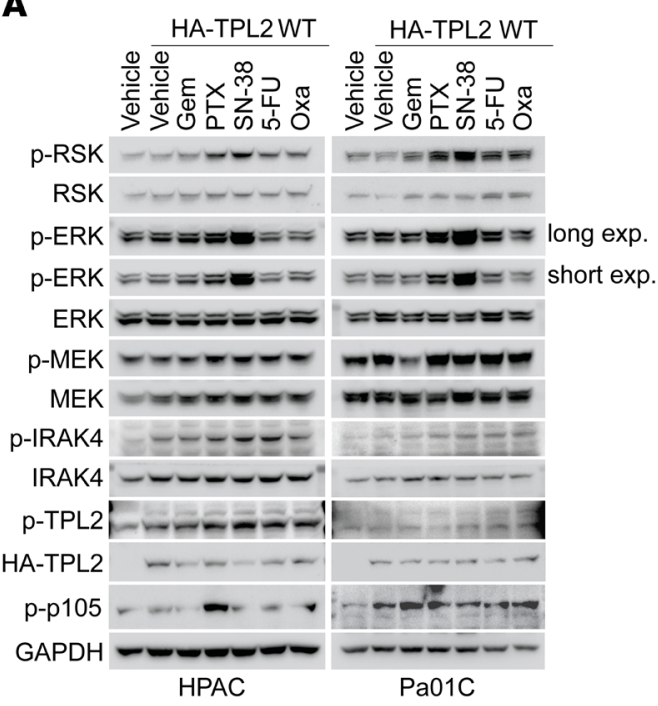

C
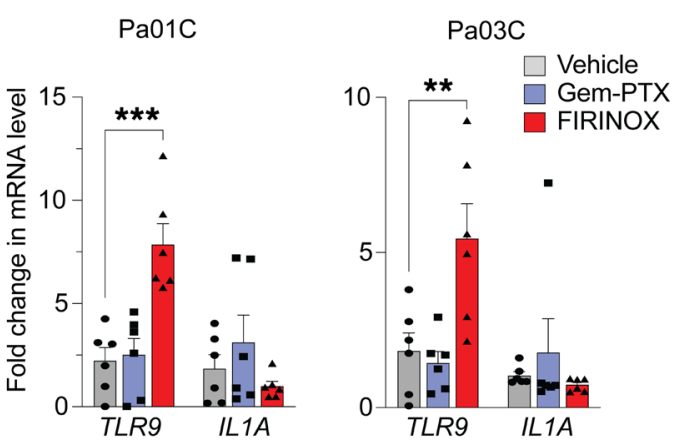

$\mathbf{E}$

$+\mathrm{SN}-38$

$+\mathrm{SN}-38$

$+\mathrm{SN}-38$

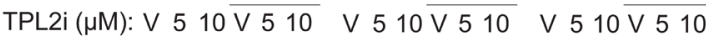

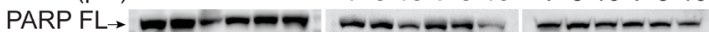
PARP cleaved $\rightarrow$, Cleaved/uncleaved:1.0 1.31.71.12.32.9 1.01 .42 .316 .715 .9191 .01 .12 .04 .84 .56 .7
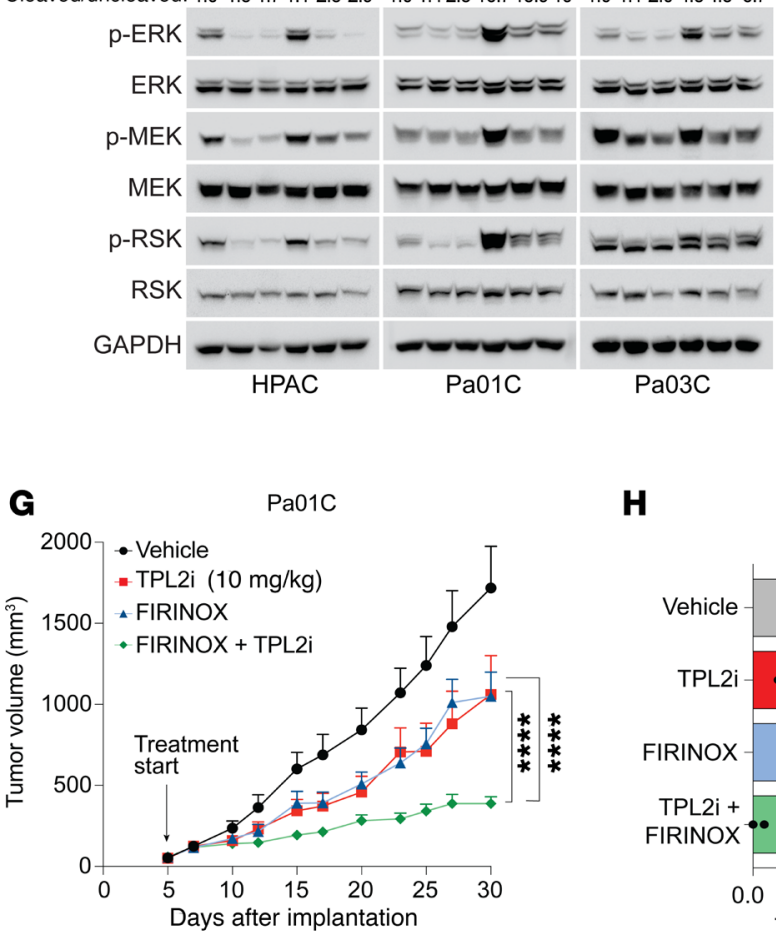

B

D
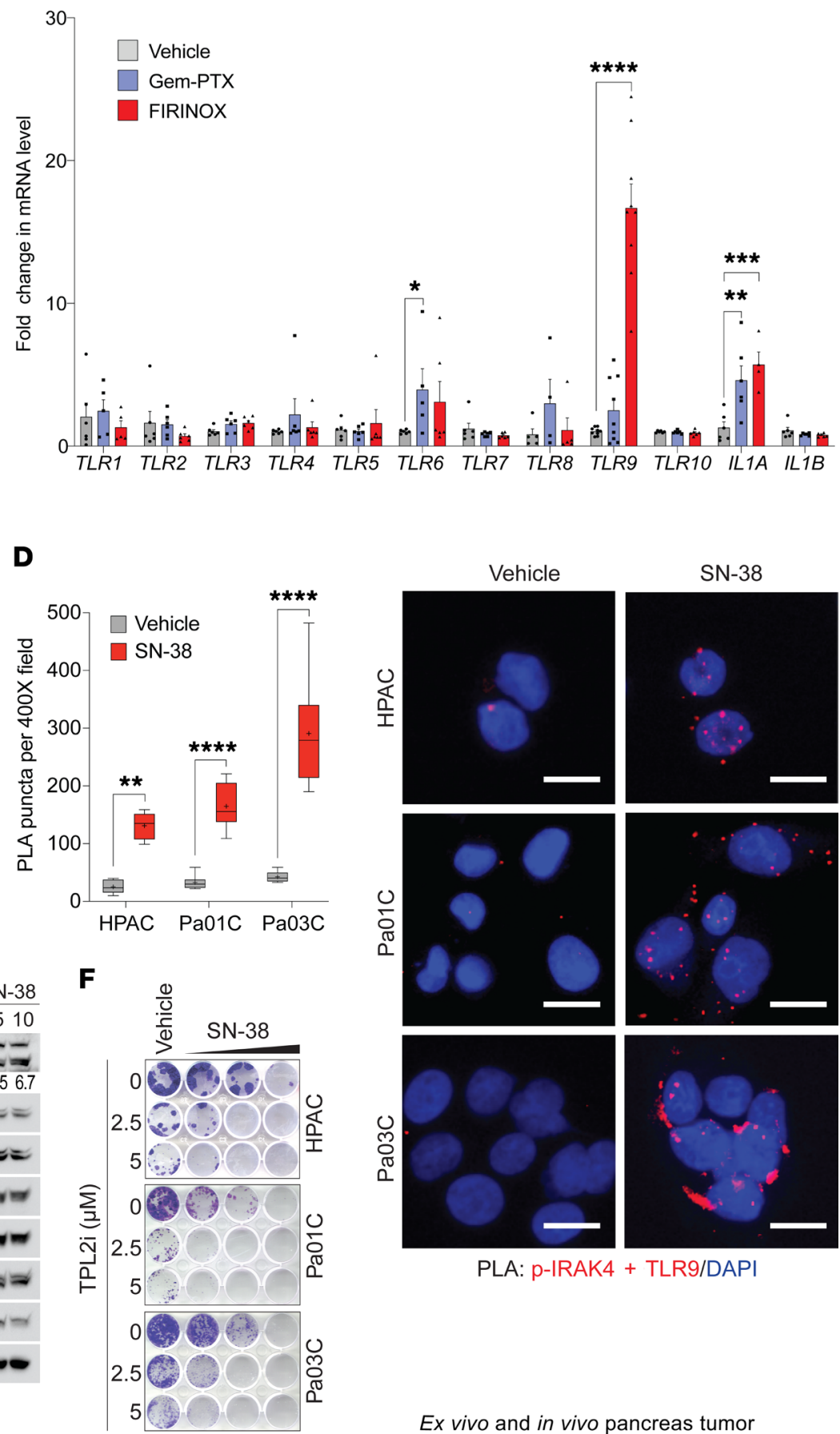

PLA: p-IRAK4 + TLR9/DAPI

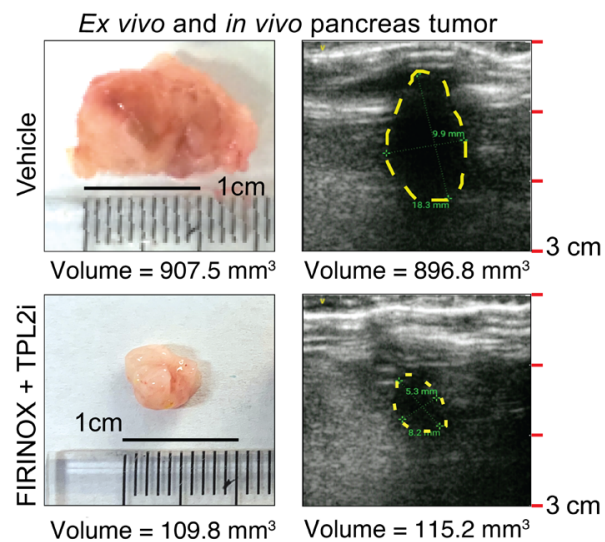

HPAC
H

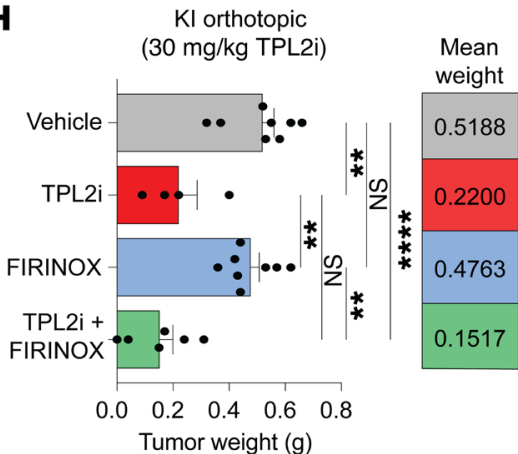


Figure 7. TPL2 inhibition potentiates chemotherapy by curbing MAPK and NF-KB activation. (A) Immunoblots of 2 PDAC cell lines overexpressing HA epitope-tagged TPL2 WT treated for 16 hours with different chemotherapy agents (10 $\mu \mathrm{M}$ each). (B and C) Quantification of mRNA transcript levels for multiple genes in 3 PDAC cell lines treated with vehicle, gemcitabine-paclitaxel (Gem-PTX), or FIRINOX (10 $\mu \mathrm{M}$ each of 5-FU, SN-38, and oxaliplatin). (D) Duolink proximity ligation assay (PLA) identifying interaction between $\mathrm{p}$-IRAK4 and TLR9 in 3 PDAC cell lines treated with $10 \mu \mathrm{M}$ SN-38 for 16 hours. Six $\times 400$ magnification fields per condition were analyzed. Scale bars: $10 \mu \mathrm{m}$. (E) Immunoblots of 3 PDAC cell lines treated with TPL2i, SN-38, or their combination for 16 hours. $\mathrm{FL}$, full length; $\mathrm{V}$, vehicle. (F) $2 \mathrm{D}$ crystal violet clonogenic colony-forming assays of 3 PDAC cell lines treated with TPL2i, SN-38 $(2.5 \mathrm{nM}, 5 \mathrm{nM}$, and $10 \mathrm{nM}$ ), or their combination in a dose matrix for 3 to 4 weeks. Data show 1 independent experiment out of $\geq 3$ per cell line. (C) Tumor volume curves for patient-derived Pa01C PDAC cells implanted subcutaneously into nude mice followed by treatment with TPL2i, FIRINOX, or combination therapy. Data represent 10 independent tumors $(n=10)$ for each drug treatment group and 8 independent tumors $(n=8)$ for vehicle-treated group. $P$ value from 2-way ANOVA followed by Tukey's multiple-comparisons test. One outlier was removed by Grubb's test, $\alpha=0.01$. (H) Graph depicting final tumor weight (final pancreas weight - $0.1 \mathrm{~g}$ [i.e., normal pancreas weight]) after orthoptic injection of murine KI cells and treatment as indicated for 14 days. Images of ex vivo and in vivo tumors detected by ultrasound along with tumor volume are shown on right. $P$ values from 1-way ANOVA with Tukey's multiple-comparisons test. All data presented as mean \pm SEM ${ }^{* * * *} P<0.0001 ;{ }^{* * *} P<0.0002 ;{ }^{* *} P<0.0021 ;{ }^{*} P<0.0332$

alter the entire protein is unclear. It has been suggested that the C-terminus of TPL2 negatively regulates TPL2 kinase activity via intramolecular interaction with its kinase domain (38). In addition, the C-terminus of TPL2, upon IKK-dependent phosphorylation of S400 and S443, binds to the 14-3-3 complex that stabilizes TPL2 and increases its kinase activity toward MEK1, possibly by relieving the kinase-inhibitory interaction between the C-terminus and kinase domain (35). The R442H mutation may impact TPL2 binding with 14-3-3, resulting in the increased stability and increased MEK and ERK activation that we observed in our experiments. On the other hand, R442 is part of a conserved MAPK recognition motif (KRQRSLYIDL) present on TPL2 (39). This raises the possibility that mutation of Arg to His at this residue may alter TPL2's binding affinity and/or specificity for substrates, although detailed additional work is required to prove this. The mechanism by which the E188K mutation stabilizes the protein is intriguing, as this residue is located within the kinase domain which is distant from the C-terminal degron. It is possible that this E188K mutation enhances the intrinsic kinase activity in addition to disrupting its degradation, but this will require resolution of the entire TPL2 protein structure. Furthermore, the mechanism including the E3 ligase that governs the degradation of TPL2 is unknown and should be investigated. This is especially important because our PDAC TMA showed p-IRAK4 level to be strongly associated with high TPL2 protein level, which in turn is associated with poor prognosis. Therefore, we speculate that high IRAK4 activity may protect TPL2 from degradation, although the detailed mechanism remains to be determined. Whether upstream TLR/IL-1R activation is required for the enhanced activity of the E188K or R442H mutant is uncertain. However, this is unlikely because these mutants exhibit markedly enhanced activity in unperturbed 293T cells compared with the WT counterpart. Importantly, both E188K and $\mathrm{R} 442 \mathrm{H}$ mutants remain sensitive to the TPL2i that we tested. TPL2i and RAFi cooperate to curb the growth of Hs695T cells that harbor MAP3K $8^{E 188 K}$ and $B R A F^{V 600 E}$ mutations, and MAPK activity in IGROV1 ovarian cancer cells that harbor only the $M A P 3 K 8^{R 442 H}$ mutation is completely abolished by low-dose TPL $2 \mathrm{i}$.

In this study, we demonstrated the in vivo antitumor efficacy of TPL $2 \mathrm{i}$ as a single agent and in combination with chemotherapy. Targeting IRAK4 or TPL2 is not expected to have severe side effects and none were observed in mice. Irak4-knockout mice are viable and have normal lifespan but are immunocompromised (33). Humans with inborn IRAK4 deficiency are susceptible to life-threatening bacterial infection in early infancy, but with proper antibiotic prophylaxis have survived into adolescence and adulthood (56). The IRAK4 inhibitor CA-4948 is now in clinical trial (NCT03328078) for patients with refractory hematologic malignancies, and is found to be rather well tolerated, with $23 \%$ of patients developing grade 1-2 neutropenia (57). Similarly, Map3k8-knockout mice do not exhibit obvious phenotypic defects, and have normal bone marrow but are impaired in MEK/ERK activation and TNF- $\alpha$ production following LPS challenge (58). To date, no TPL2i has been developed and tested in clinical trials. We argue that TPL2 is a more versatile kinase that controls multiple oncogenic pathways besides MEK/ERK, and therefore development of a dedicated TPL2i is needed especially for KRAS- or MAP3K8-mutant cancers.

In conclusion, our study comprehensively describes an essential role of IRAK4 and TPL2 in oncogenic RAS signaling, using PDAC as a disease model. Mechanistically, we show that the IRAK4/TPL2 axis is differentially engaged in the basal state versus during genotoxic stress by different upstream receptors. We show that TPL2 inhibition synergistically sensitizes PDAC to chemotherapy in in vivo models, which we propose is a potentially novel therapeutic strategy. Finally, we characterize 2 gain-of-function mutations of MAP3K8 (TPL2) in melanoma and ovarian cancer, which complement other studies describing overexpression, truncations, or fusion of MAP3K8 (TPL2) as being oncogenic. Overall, our study urges development of dedicated TPL2is and detection of MAP3K8 (TPL2) mutations for cancer patients.

\section{Methods}

Additional methods description can be found in Supplemental Methods.

Cell lines. All cell lines including HPNE, HPNE-KRAS $S^{G 12 D}$, HPAC, and Hs695T were obtained from ATCC, which performed its own authentication by short tandem repeat DNA profiling. IGROV1 cells were a gift from Katherine Fuh (Washington University, St. Louis, Missouri, USA), originated from the NCI-60 panel (59) and not further authenticated. The HEK T/tH cell line was a gift from Christopher Counter (Duke University, Durham, North Carolina, USA) and previously published (60). The KP2 cell line was a gift from David DeNardo (Washington University) and authenticated by whole-exome sequencing (61). MEFs were isolated from WT or IRAK4-null mice, as described previously (62). The patient-derived cell lines $\mathrm{Pa} 01 \mathrm{C}, \mathrm{Pa} 02 \mathrm{C}, \mathrm{PaO3C}$, $\mathrm{Pa} 04 \mathrm{C}, \mathrm{Pa} 14 \mathrm{C}$, and Pa16C were a gift from Channing J. Der (University of North Carolina, Chapel Hill, North Carolina, USA) and have been described previously (63). All cell lines were cultured in DMEM supplemented with $10 \%$ fetal bovine serum and $1 \%$ penicillin/streptomycin except IGROV1 and Hs695T, which were cultured in RPMI-1640 and 
A

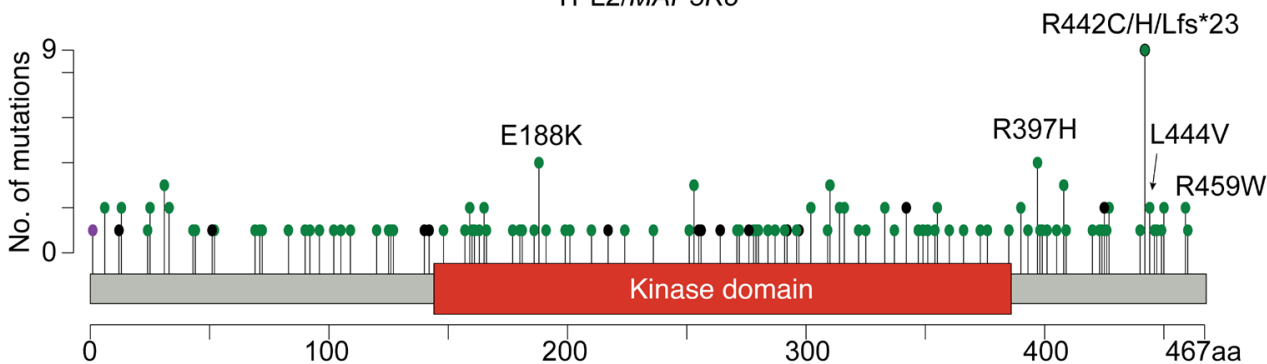

B

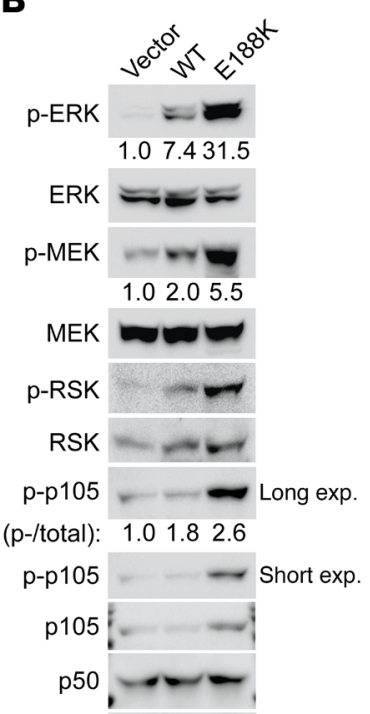

C

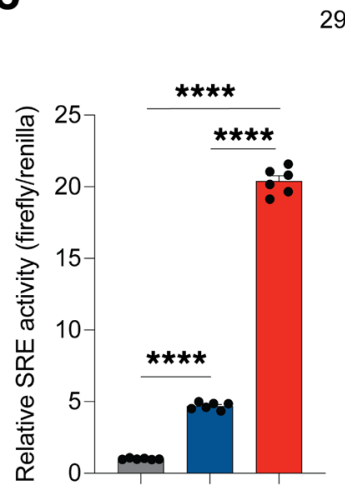

EV WT E188K

HA-TPL

GAPDH
293T

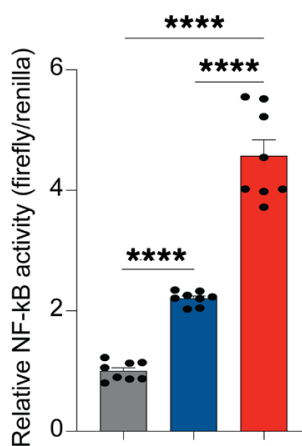

EV WT E188K

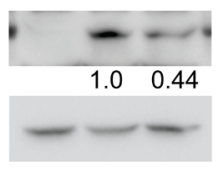

D

Relative expression $\left(\log _{2}\right)$

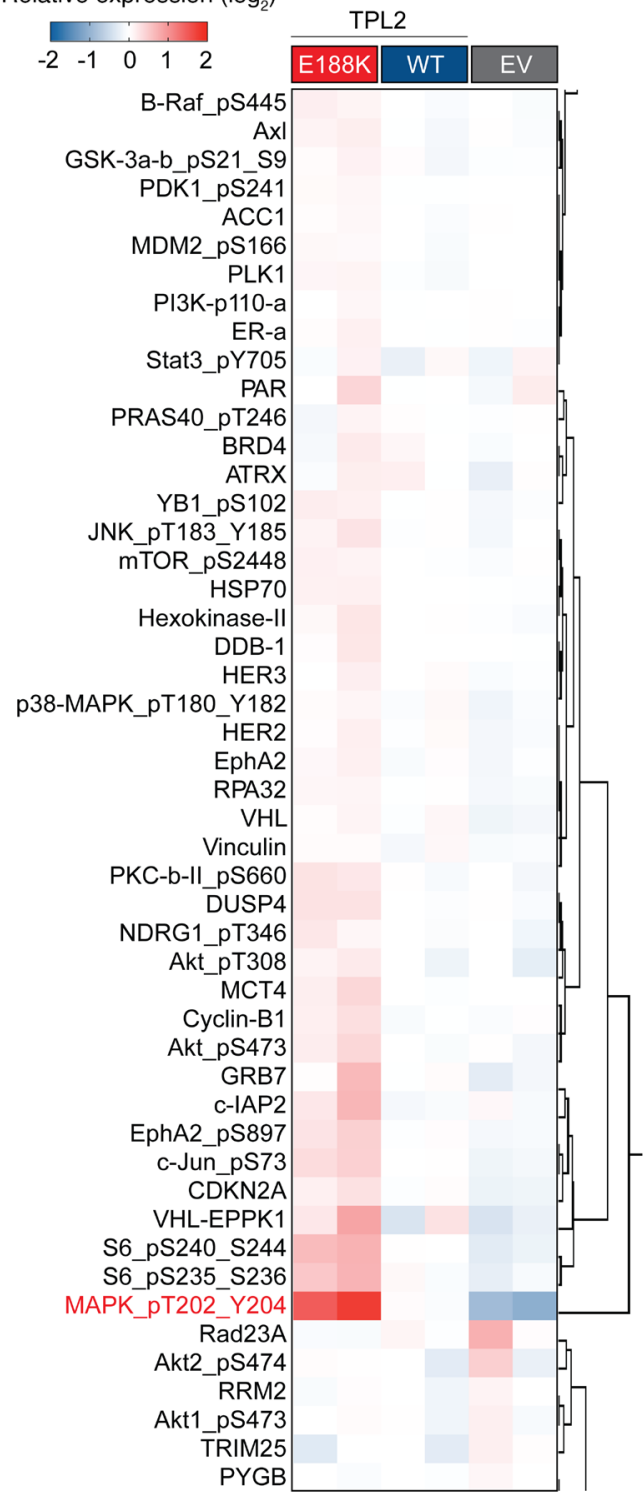

E

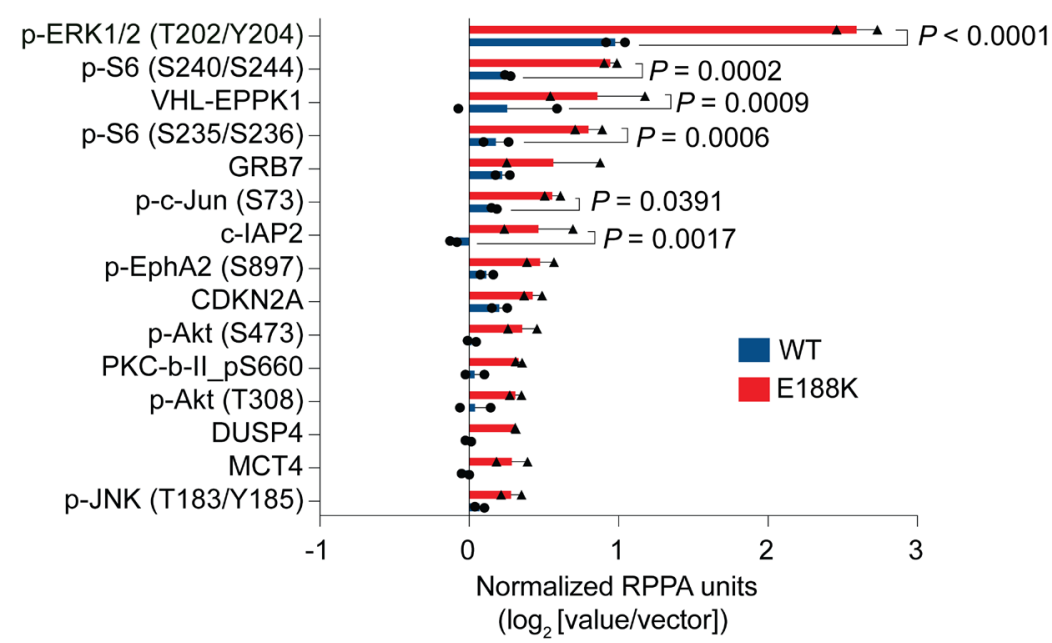

RPPA: top 15 upregulated markers

$\log _{2}$ [value/vector])

Figure 8. E188K mutation in MAP3K8/TPL2 is a gain-of-function mutation. (A) Lollipop plot adapted from CBioPortal.org (as of August 2019) identifying mutations in MAP3K8/TPL2. (B) Immunoblots of $293 \mathrm{~T}$ cells transfected with empty expression vector (EV, control) or vector encoding HA epitope-tagged WT TPL2 or TPL2 E188K for 48 hours. (C) Relative serum-response element (SRE) activity and NF- $\kappa$ B reporter activity in 293T cells transfected as in B. Data are from 2 independent experiments. ${ }^{* * *} P<0.0001$ by 2 -way ANOVA followed by Tukey's multiple-comparisons test. (D) Heatmap representing relative expression in $\log _{2}$ units of proteins evaluated by reverse-phase protein array (RPPA) of 293T cells transfected with empty vector (EV, control) or vector encoding HA-tagged WT TPL2 or TPL2 E188K in duplicate $(n=2)$ for 48 hours. (E) Relative abundance (in $\log _{2}$ units) of the top 15 upregulated targets in RPPA shown in D. p-ERK (in bold text) is the top hit and significantly upregulated targets are indicated with $P$ values from 2-way ANOVA with Tukey's multiple-comparisons test. All data presented as mean \pm SEM. 
A

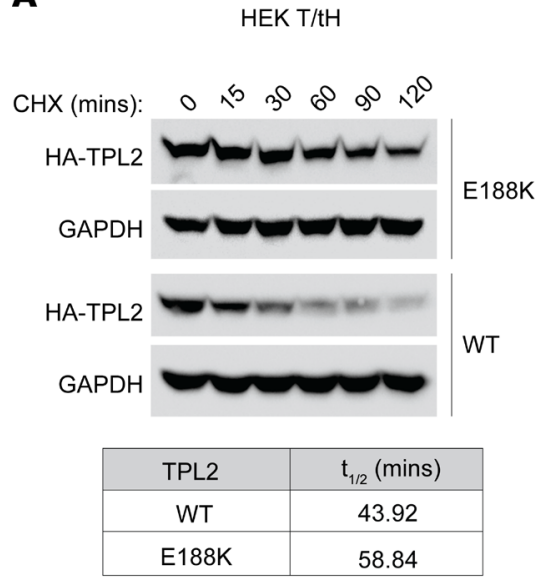

B

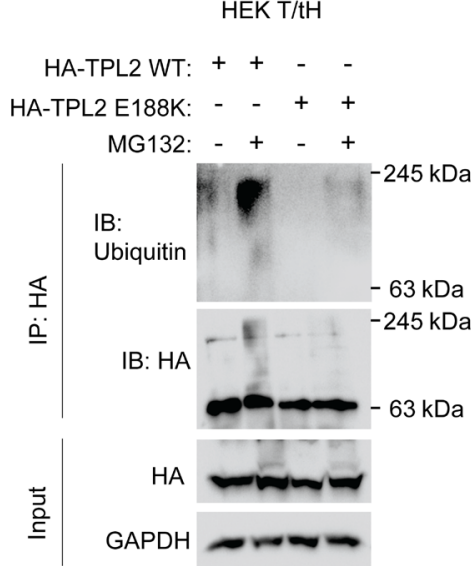

C

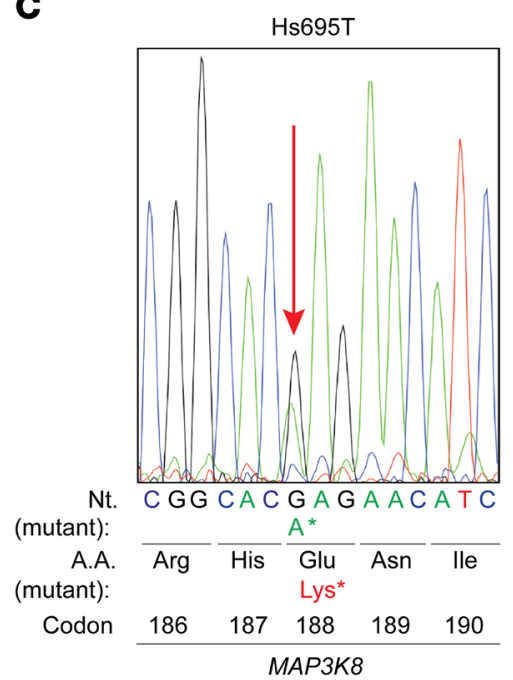

\section{Hs695T}

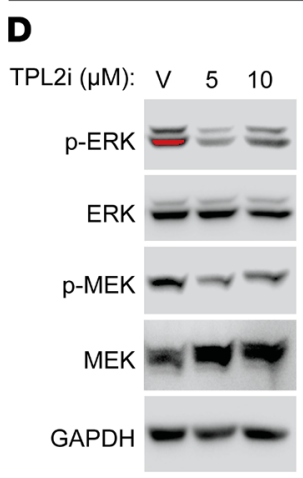

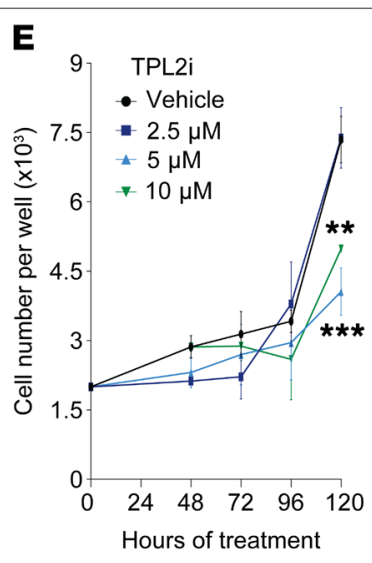

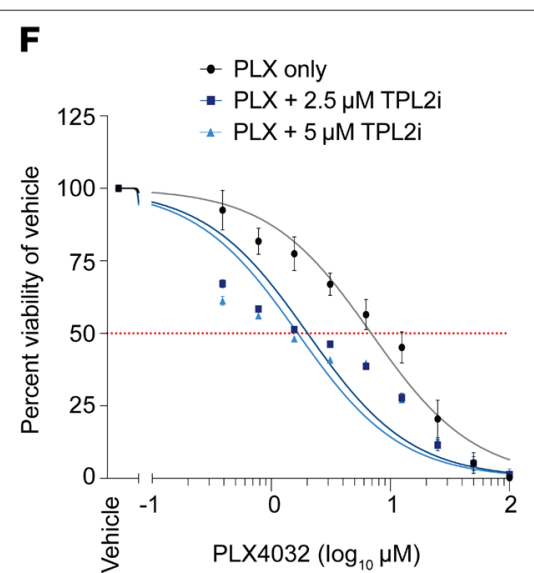

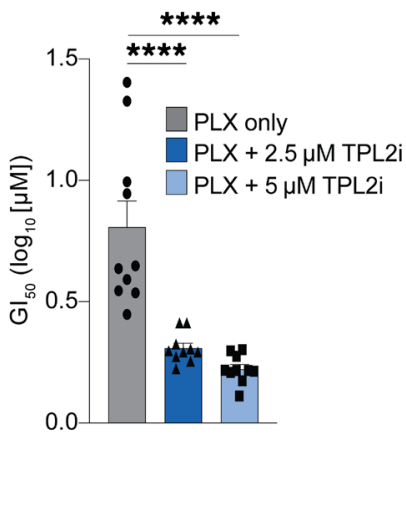

Figure 9. E188K mutation stabilizes TPL2 protein. (A) Immunoblots of HEK T/tH cells stably expressing HA epitope-tagged WT TPL2 or TPL2 E188K treated with $10 \mu \mathrm{g} / \mathrm{mL}$ cycloheximide (CHX). Table below shows half-life ( $\left.\mathrm{t}_{1 / 2}\right)$ of WT TPL2 and TPL2 E188K protein calculated by measuring HA-TPL2 band intensities, normalizing to $t_{0}$ and performing 1-phase exponential decay. Data represent 1 of 3 independent experiments showing similar results. (B) Immunoprecipitation of HA epitope-tagged WT TPL2 or TPL2 E188K treated with $10 \mu$ M MG132 (proteasome inhibitor) and immunoblotted as indicated. (C) Sanger sequencing peaks of MAP3K8 (TPL2) locus in Hs695T. Arrow indicates the naturally occurring missense point mutation responsible for the $\mathrm{Clu} \rightarrow$ Lys substitution at codon 188 (E188K) in TPL2 in the Hs695T cell line. (D) Immunoblot of Hs695T cells treated with TPL2i or vehicle (V) for 24 hours in $10 \%$ serum-containing media. (E) Proliferation of Hs695T cells treated with TPL2i. P values from 2-way ANOVA with Dunnett's multiple-comparisons test. (F) Viability of Hs695T cells treated with PLX4032 (BRAFi) alone or in combination with 2 different concentrations of TPL2i. Graph on right depicts GI ${ }_{50}$ of PLX4032 in $\log _{10}$ units. Data represent 10 replicates from 4 independent experiments. $P$ values are from 1-way ANOVA with Dunnett's multiple-comparisons test. All data presented as mean $\pm \mathrm{SEM}$. ${ }^{* * *} P<0.0001 ;{ }^{* *} P<0.0002 ;{ }^{* *} P<0.0021$.

MEM, respectively, with nonessential amino acids along with other supplements stated above. Mycoplasma testing was performed annually using a MycoSEQ Detection kit (Applied Biosystems). All lines were used for fewer than 6 months after receipt or resuscitation from cryopreservation. For all drug treatments, when applicable, a concentration of 0 (zero) is treatment with vehicle, DMSO.

In vivo tumorigenesis assays. For subcutaneous xenograft, approximately 5 million cells per flank were implanted into 6-week-old athymic nude mice (NU/J, Jackson Laboratory). When applicable, treatment with drug compound was initiated when tumors were palpable. FIRINOX (25 mg/kg 5-FU, $17.5 \mathrm{mg} / \mathrm{kg}$ irinotecan, and $3.35 \mathrm{mg} / \mathrm{kg}$ oxaliplatin) was administered via intraperitoneal injection weekly in $50 \mu \mathrm{L}$ PBS. TPL2i was administered by intraperitoneal injection at 10 $\mathrm{mg} / \mathrm{kg}$ in $40 \mu \mathrm{L} \mathrm{DMSO}, 5$ days per week. Mice in the control group were treated with vehicle. Tumor volume was calculated as width ${ }^{2} \times$ (length $\times 0.5$ ). Tumors were measured and mice were weighed 3 days per week. For orthotopic implantation, murine KI PDAC cells were injected into the pancreas of 7-week-old female FVB/NJ mice (Jackson Laboratory), as previously described (64). Six days after implantation, treatment with vehicle, FIRINOX (same dose as above), or TPL2i (30 $\mathrm{mg} / \mathrm{kg}$ ) was initiated for 14 days, at which time all mice were sacrificed. In vivo tumor progression was monitored using ultrasound (VScan, GE Healthcare) with final day representative tumor images shown.

Statistics. All results, when applicable, are expressed as mean \pm SEM. Statistical analysis was performed using GraphPad Prism v7 or v8 software. Unpaired, 2-tailed (2-sided) Student's $t$ tests were used to compare 2 groups when appropriate. For multiple groups, 1-way or 2-way ANOVA with appropriate post hoc test was used. In instances 
A
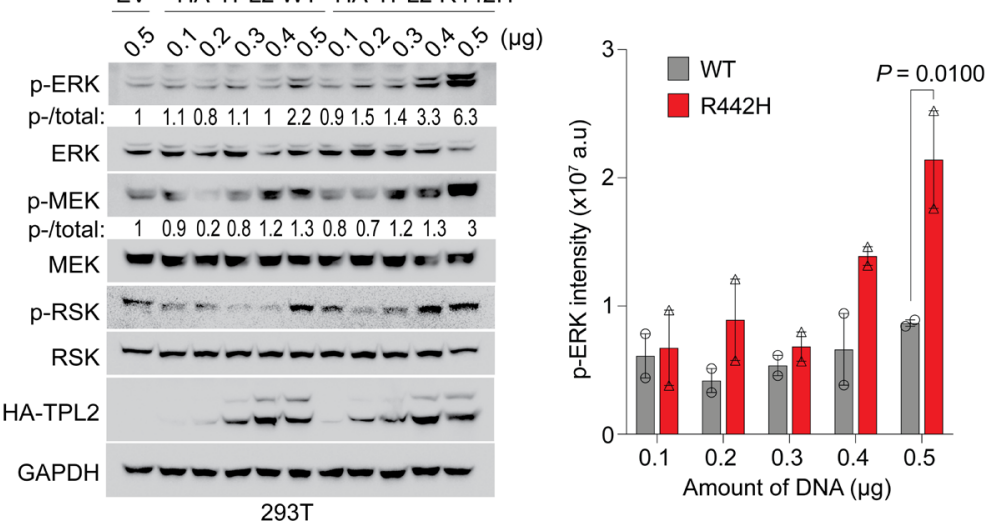

C

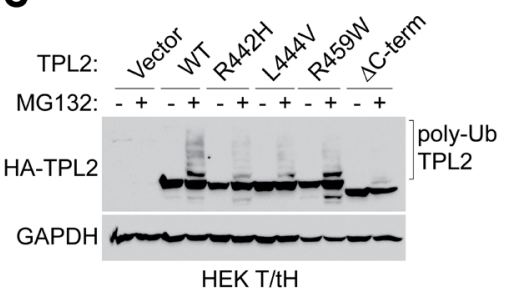

D

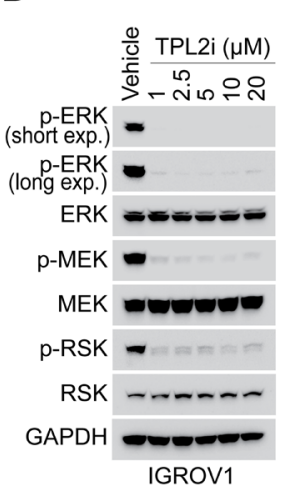

B

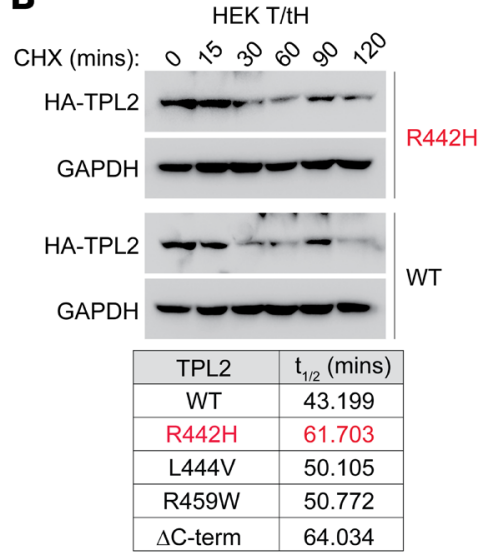

E

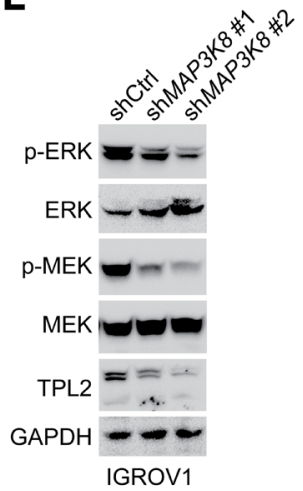

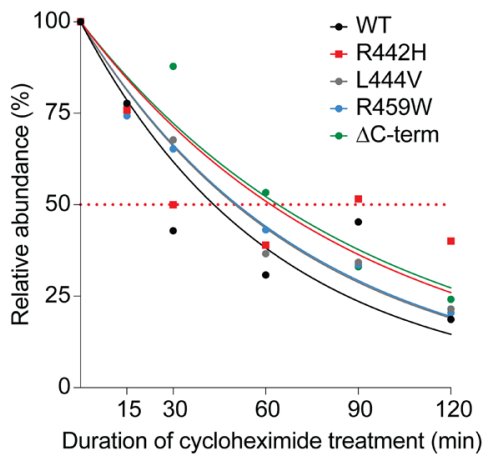

IGROV1
$\mathbf{F}$

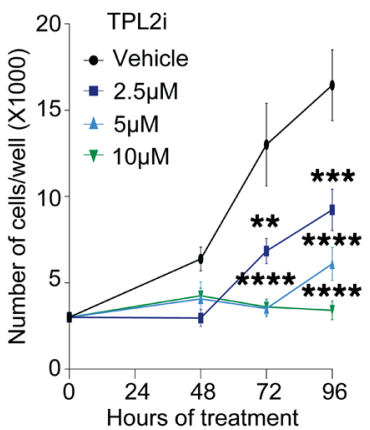

G

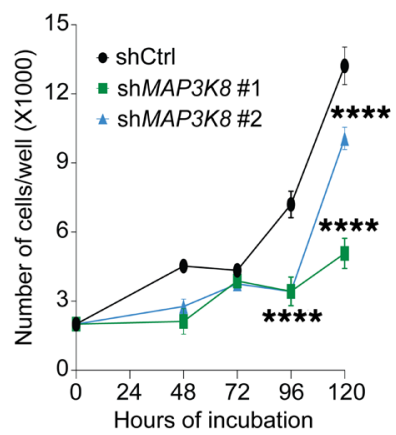

IGROV1

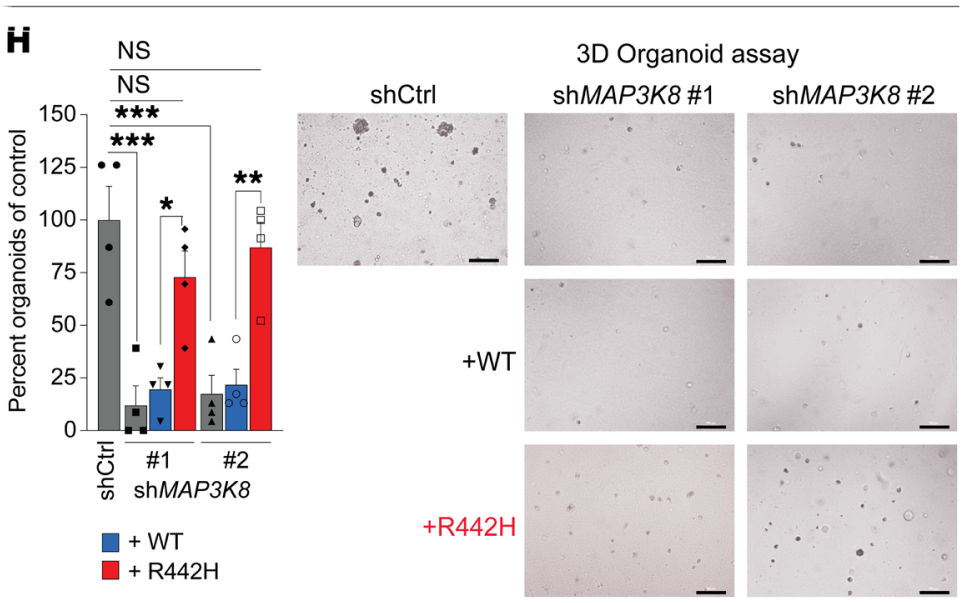

Figure 10. R442H is a gain-of-function mutation that curtails proteasomal degradation of TPL2. (A) Immunoblots of 293T cells transiently transfected with empty vector (EV), or vector encoding HA epitope-tagged WT TPL2 or TPL2 R442H for 48 hours. On right is quantification of $\mathrm{p}$-ERK band intensities for WT TPL2 and TPL2 R442H samples from immunoblots on left. Data represent 2 independent experiments. $P$ values from 2-way ANOVA with Holm-Šidák multiple-comparisons test. (B) Immunoblots of HEK T/tH cells stably expressing vector encoding WT TPL2 or TPL2 R442H and treated with $10 \mu \mathrm{g} / \mathrm{mL}$ cycloheximide (CHX) for indicated durations. Table below states half-life $\left(t_{1 / 2}\right)$ of TPL2 WT and mutant proteins calculated by measuring HA-TPL2 band intensities, normalizing to $t_{0}$ and performing 1-phase exponential decay analysis as shown in graph at the bottom. Experiment was performed 3 times and 1 set of data is shown. (C) Immunoblots of HEK T/tH cells stably expressing empty vector or HA epitope-tagged TPL2 mutants treated with 10 M MG132 (proteasome inhibitor) for 4 hours. One of 2 or more independent experiments is shown. C-terminally truncated TPL2 $(\Delta C$-term) is used as positive control. Poly-Ub, polyubiquitinated. (D) Immunoblots of IGROV1 cells serum starved for 24 hours and treated with TPL2i for 2 hours. (E) Immunoblots of IGROV1 cells after shRNA-mediated TPL2 depletion. ( $F$ and $\mathbf{G}$ ) Proliferation of IGROV1 cells treated with TPL2i and after shRNA-mediated TPL2 depletion, respectively. $P$ values from 2-way ANOVA with Dunnett's multiple-comparisons test. $(\mathbf{H}) 3 \mathrm{D}$ organoid growth of IGROV1 cells after shRNA-mediated TPL2 depletion and rescue with either WT TPL2 or R442H mutant. Organoids were counted from 4 independent transfected wells per condition. Scale bars: $100 \mu \mathrm{m}$. $P$ values from 1-way ANOVA with Dunnett's multiplecomparisons test. All data presented as mean $\pm \mathrm{SEM}$. ${ }^{* * * *} P<$ $0.0001 ;{ }^{* *} P<0.0002 ;{ }^{* *} P<0.0021 ;{ }^{*} P<0.0332$. 
of systemic/group variation, repeated-measures ANOVA was used. Unadjusted $P$ values less than 0.05 were considered statistically significant. Adjusted $P$ value metrics are stated at end of each figure legend where applicable. Cox proportional hazards models were used to evaluate the relationships between clinical characteristics and overall survival. Kaplan-Meier curve was generated using SAS version 9.4 (SAS Institute) and analyzed by log-rank tests.

Data availability. RNA sequencing data on KP2 WT, Irak4-knockout and -rescue cells were deposited in NCBI's Gene Expression Omnibus (GEO), accession number GSE148442. Complete, unedited Western blot images are provided in the supplemental material. All sgRNA and shRNA sequences, and qRT-PCR primers are listed in Supplemental Tables 4 and 5, respectively.

Study approval. The Washington University PDAC TMA was IRB approved (no. 201404143) and previously published (65). Patient consent was waived per IRB approval. All studies were performed per ethical principles of the Declaration of Helsinki. All animal (mouse) experiments were conducted under IACUC approval (no. 20190138).

\section{Author contributions}

PBD with KHL developed the project, designed the study, and wrote the manuscript. PBD performed most experiments, completed all revisions, and acquired and analyzed most of the data, including but not limited to generating cell lines, cloning TPL2 mutant plasmid constructs, Western blotting, mouse experiments, qPCR, in vitro growth assays, bioinformatics analyses, and IHC H-score analysis. NK performed Western blotting to evaluate degradation of TPL2 C-terminal mutants. DZ, HJ, YC, and LL each contributed one or more panels of data in the manuscript, including DuoLink assay, soft agar, and mouse experiments with IRAK4-knockout cells, flow cytometry, and IHC staining, respectively. LL, QW, KS, and PMG provided technical assistance. AWG generated the human PDAC TMA. KHL assigned author responsibilities, supervised, provided funding, and administered the project.

\section{Acknowledgments}

This study was supported by the NIH (R37CA219697-01), a Washington University SPORE Career Enhancement Award grant (1P50CA196510-01A1), the American Cancer Society (RSG-17-203-01-TBG), and the Alvin J. Siteman Cancer Center Siteman Investment Program (supported by the Barnard Trust and The Foundation for Barnes-Jewish Hospital). We acknowledge the Washington University Digestive Disease Research Core Center (DDRCC) (NIH grant P30 DK052574) for providing technical support. The content is solely the responsibility of the authors and does not necessarily represent the official view of the NIH.

Address correspondence to: Kian-Huat Lim, Washington University School of Medicine, 660 South Euclid Avenue, Campus Box 8069, Saint Louis, Missouri 63110, USA. Phone: 314.362.6157; Email: kian-huat.lim@wustl.edu.
1. Canon J, et al. The clinical KRAS(G12C) inhibitor AMG 510 drives anti-tumour immunity. Nature. 2019;575(7781):217-223.

2. Waters AM, Der CJ. KRAS: the critical driver and therapeutic target for pancreatic cancer. Cold Spring Harb Perspect Med. 2018;8(9):a031435.

3. Chung V, et al. Effect of selumetinib and MK-2206 vs oxaliplatin and fluorouracil in patients with metastatic pancreatic cancer after prior therapy: SWOG S1115 study randomized clinical trial. JAMA Oncol. 2017;3(4):516-522.

4. Algül H, Adler G, Schmid RM. NF-kappaB/ Rel transcriptional pathway: implications in pancreatic cancer. Int J Gastrointest Cancer. 2002;31(1-3):71-78.

5. Wang W, Abbruzzese JL, Evans DB, Larry L, Cleary KR, Chiao PJ. The nuclear factor-kappa B RelA transcription factor is constitutively activated in human pancreatic adenocarcinoma cells. Clin Cancer Res. 1999;5(1):119-127.

6. Prabhu L, Mundade R, Korc M, Loehrer PJ, $\mathrm{Lu}$ T. Critical role of NF- $\kappa \mathrm{B}$ in pancreatic cancer. Oncotarget. 2014;5(22):10969-10975.

7. Zhang D, et al. Constitutive IRAK4 activation underlies poor prognosis and chemoresistance in pancreatic ductal adenocarcinoma. Clin Cancer Res. 2017;23(7):1748-1759.

8. Ling J, et al. KrasG12D-induced IKK2/ $\beta / \mathrm{NF}-\kappa \mathrm{B}$ activation by IL- $1 \alpha$ and $\mathrm{p} 62$ feedforward loops is required for development of pancreatic ductal adenocarcinoma. Cancer Cell. 2012;21(1):105-120.

9. Prescott JA, Cook SJ. Targeting IKK $\beta$ in cancer: challenges and opportunities for the therapeutic utilisation of IKK $\beta$ inhibitors. Cells. 2018;7(9):E115.
10. Zhang D, et al. Tumor-stroma IL1 $\beta$-IRAK4 feedforward circuitry drives tumor fibrosis, chemoresistance, and poor prognosis in pancreatic cancer. Cancer Res. 2018;78(7):1700-1712.

11. Jain A, Kaczanowska S, Davila E. IL-1 receptorassociated kinase signaling and its role in inflammation, cancer progression, and therapy resistance. Front Immunol. 2014;5:553.

12. Lim KH, Staudt LM. Toll-like receptor signaling. Cold Spring Harb Perspect Biol. 2013;5(1):a011247.

13. Srivastava R, et al. Augmentation of therapeutic responses in melanoma by inhibition of IRAK-1,4. Cancer Res. 2012;72(23):6209-6216.

14. Wee ZN, et al. IRAK1 is a therapeutic target that drives breast cancer metastasis and resistance to paclitaxel. Nat Commun. 2015;6:8746.

15. Adams AK, et al. IRAK1 is a novel DEK transcriptional target and is essential for head and neck cancer cell survival. Oncotarget. 2015;6(41):43395-43407.

16. Li Q, et al. IRAK4 mediates colitis-induced tumorigenesis and chemoresistance in colorectal cancer. JCI Insight. 2019;4(19):130867.

17. Weichert W, et al. High expression of RelA/p65 is associated with activation of nuclear factorkappaB-dependent signaling in pancreatic cancer and marks a patient population with poor prognosis. Br J Cancer. 2007;97(4):523-530.

18. Fujioka $S$, et al. Function of nuclear factor kappaB in pancreatic cancer metastasis. Clin Cancer Res. 2003;9(1):346-354.

19. Liberzon A, Birger C, Thorvaldsdóttir H, Ghandi M, Mesirov JP, Tamayo P. The Molecular Signatures Database (MSigDB) hallmark gene set collection. Cell Syst. 2015;1(6):417-425.
20. Lin SC, Lo YC, Wu H. Helical assembly in the MyD88-IRAK4-IRAK2 complex in TLR/IL-1R signalling. Nature. 2010;465(7300):885-890.

21. Adachi O, et al. Targeted disruption of the MyD88 gene results in loss of IL-1- and IL-18mediated function. Immunity. 1998;9(1):143-150.

22. Zhuang Z, et al. IL1 receptor antagonist inhibits pancreatic cancer growth by abrogating NF- $\kappa \mathrm{B}$ activation. Clin Cancer Res. 2016;22(6):1432-1444.

23. Chien Y, et al. RalB GTPase-mediated activation of the IkappaB family kinase TBK1 couples innate immune signaling to tumor cell survival. Cell. 2006;127(1):157-170.

24. Dan HC, Cooper MJ, Cogswell PC, Duncan JA, Ting JP, Baldwin AS. Akt-dependent regulation of NF-\{kappa\}B is controlled by mTOR and Raptor in association with IKK. Genes Dev. 2008;22(11):1490-1500.

25. Lee KL, et al. Discovery of clinical candidate 1-\{[(2S,3S,4S)-3-ethyl-4-fluoro-5-oxopyrrolidin-2-yl]methoxy\}-7-methoxyisoquinoline-6-carboxamide (PF-06650833), a potent, selective inhibitor of interleukin-1 receptor associated kinase 4 (IRAK4), by fragment-based drug design. J Med Chem. 2017;60(13):5521-5542.

26. Mielke LA, et al. Tumor progression locus 2 (Map3k8) is critical for host defense against Listeria monocytogenes and IL-1 beta production. J Immunol. 2009;183(12):7984-7993.

27. Pattison MJ, et al. TLR and TNF-R1 activation of the MKK3/MKK6-p38 $\alpha$ axis in macrophages is mediated by TPL-2 kinase. Biochem J. 2016;473(18):2845-2861.

28. Babu GR, et al. Phosphorylation of NF-kappaB1/ p105 by oncoprotein kinase Tpl2: implications 
for a novel mechanism of Tpl2 regulation. Biochim Biophys Acta. 2006;1763(2):174-181.

29. Gavrin LK, et al. Inhibition of Tpl2 kinase and TNF-alpha production with 1,7-naphthyridine-3-carbonitriles: synthesis and structureactivity relationships. Bioorg Med Chem Lett. 2005;15(23):5288-5292.

30. Das S, et al. Tpl2/cot signals activate ERK, JNK, and NF-kappaB in a cell-type and stimulus-specific manner.J Biol Chem. 2005;280(25):23748-23757.

31. Conroy T, et al. FOLFIRINOX versus gemcitabine for metastatic pancreatic cancer. $N$ EnglJ Med. 2011;364(19):1817-1825.

32. Von Hoff DD, et al. Increased survival in pancreatic cancer with nab-paclitaxel plus gemcitabine. N Engl JMed. 2013;369(18):1691-1703.

33. Suzuki N, et al. Severe impairment of interleukin-1 and Toll-like receptor signalling in mice lacking IRAK-4. Nature. 2002;416(6882):750-756.

34. Beinke S, Robinson MJ, Hugunin M, Ley SC. Lipopolysaccharide activation of the TPL-2/ MEK/extracellular signal-regulated kinase mitogen-activated protein kinase cascade is regulated by IkappaB kinase-induced proteolysis of NF-kappaB1 p105. Mol Cell Biol. 2004;24(21):9658-9667.

35. Ben-Addi A, et al. IкB kinase-induced interaction of TPL-2 kinase with 14-3-3 is essential for Toll-like receptor activation of ERK-1 and -2 MAP kinases. Proc Natl Acad Sci U S A. 2014;111(23):E2394-E2403.

36 . Roget $\mathrm{K}$, et al. I $\mathrm{B}$ kinase 2 regulates TPL-2 activation of extracellular signal-regulated kinases 1 and 2 by direct phosphorylation of TPL- 2 serine 400. Mol Cell Biol. 2012;32(22):4684-4690.

37. Cho J, Melnick M, Solidakis GP, Tsichlis PN. Tpl2 (tumor progression locus 2) phosphorylation at Thr290 is induced by lipopolysaccharide via an Ikappa-B Kinase-beta-dependent pathway and is required for Tpl2 activation by external signals. J Biol Chem. 2005;280(21):20442-20448.

38. Ceci JD, et al. Tpl-2 is an oncogenic kinase that is activated by carboxy-terminal truncation. Genes Dev. 1997;11(6):688-700.

39. Newman S, et al. Clinical genome sequencing uncovers potentially targetable truncations and fusions of MAP3K8 in spitzoid and other melanomas. Nat Med. 2019;25(4):597-602.

40. Ghandi M, et al. Next-generation characteri- zation of the Cancer Cell Line Encyclopedia. Nature. 2019;569(7757):503-508.

41. Gruosso T, et al. MAP3K8/TPL-2/COT is a potential predictive marker for MEK inhibitor treatment in high-grade serous ovarian carcinomas. Nat Commun. 2015;6:8583.

42. Zhu Z, et al. Inhibition of KRAS-driven tumorigenicity by interruption of an autocrine cytokine circuit. Cancer Discov. 2014;4(4):452-465.

43. Ancrile BB, O'Hayer KM, Counter CM. Oncogenic ras-induced expression of cytokines: a new target of anti-cancer therapeutics. Mol Interv. 2008;8(1):22-27.

44. Nagathihalli NS, et al. Inverse correlation of STAT3 and MEK signaling mediates resistance to RAS pathway inhibition in pancreatic cancer. Cancer Res. 2018;78(21):6235-6246.

45. Nagathihalli NS, et al. Signal transducer and activator of transcription 3 , mediated remodeling of the tumor microenvironment results in enhanced tumor drug delivery in a mouse model of pancreatic cancer. Gastroenterology. 2015;149(7):1932-1943.e9.

46. Ancrile B, Lim KH, Counter CM. Oncogenic Ras-induced secretion of IL6 is required for tumorigenesis. Genes Dev. 2007;21(14):1714-1719.

47. Senger K, et al. The kinase TPL2 activates ERK and p38 signaling to promote neutrophilic inflammation. Sci Signal. 2017;10(475):eaah4273.

48. Salaroglio IC, Mungo E, Gazzano E, Kopecka J, Riganti C. ERK is a pivotal player of chemo-immune-resistance in cancer. Int J Mol Sci. 2019;20(10):E2505.

49. Infante JR, et al. A randomised, double-blind, placebo-controlled trial of trametinib, an oral MEK inhibitor, in combination with gemcitabine for patients with untreated metastatic adenocarcinoma of the pancreas. Eur J Cancer. 2014;50(12):2072-2081.

50. Van Cutsem E, et al. Phase I/II trial of pimasertib plus gemcitabine in patients with metastatic pancreatic cancer. Int J Cancer. 2018;143(8):2053-2064.

51. Gioeli D, et al. Compensatory pathways induced by MEK inhibition are effective drug targets for combination therapy against castrationresistant prostate cancer. Mol Cancer Ther. 2011;10(9):1581-1590.

52. Johannessen CM, et al. COT drives resistance to RAF inhibition through MAP kinase pathway reactivation. Nature. 2010;468(7326):968-972.

53. Christoforidou AV, Papadaki HA, Margioris AN, Eliopoulos GD, Tsatsanis C. Expression of the Tpl2/Cot oncogene in human T-cell neoplasias. Mol Cancer. 2004;3(1):34.

54. Clark AM, Reynolds SH, Anderson M, Wiest JS. Mutational activation of the MAP3K8 protooncogene in lung cancer. Genes Chromosomes Cancer. 2004;41(2):99-108.

55. Sourvinos G, Tsatsanis C, Spandidos DA. Overexpression of the Tpl-2/Cot oncogene in human breast cancer. Oncogene. 1999;18(35):4968-4973.

56. von Bernuth H, Picard C, Puel A, Casanova JL. Experimental and natural infections in MyD88and IRAK-4-deficient mice and humans. Eur J Immunol. 2012;42(12):3126-3135.

57. Rosenthal AC, et al. Phase 1 study of CA-4948, a novel inhibitor of interleukin-1 receptorassociated kinase 4 (IRAK4) in patients (pts) with $\mathrm{r} / \mathrm{r}$ non-Hodgkin lymphoma. J Clin Oncol. 2019;37(15_suppl):e19055.

58. Dumitru CD, et al. TNF-alpha induction by LPS is regulated posttranscriptionally via a Tpl2/ERK-dependent pathway. Cell. 2000;103(7):1071-1083.

59. Shoemaker RH. The NCI60 human tumour cell line anticancer drug screen. Nat Rev Cancer. 2006;6(10):813-823.

60. Lim KH, et al. Activation of RalA is critical for Ras-induced tumorigenesis of human cells. Cancer Cell. 2005;7(6):533-545.

61. Jiang $\mathrm{H}$, et al. Targeting focal adhesion kinase renders pancreatic cancers responsive to checkpoint immunotherapy. Nat Med 2016;22(8):851-860.

62. Durkin ME, Qian X, Popescu NC, Lowy DR. Isolation of mouse embryo fibroblasts. Bio Protoc. 2013;3(18):e908.

63. Jones $\mathrm{S}$, et al. Core signaling pathways in human pancreatic cancers revealed by global genomic analyses. Science. 2008;321(5897):1801-1806.

64. Kim MP, Evans DB, Wang H, Abbruzzese JL, Fleming JB, Gallick GE. Generation of orthotopic and heterotopic human pancreatic cancer xenografts in immunodeficient mice. Nat Protoc. 2009;4(11):1670-1680.

65. Lim KH, et al. A clinically feasible multiplex proteomic immunoassay as a novel functional diagnostic for pancreatic ductal adenocarcinoma. Oncotarget. 2017;8(15):24250-24261. 